\title{
Belief Disagreements and Collateral Constraints
}

\section{Citation}

Simsek, Alp. Forthcoming. Belief disagreements and collateral constraints. Econometrica 80.

\section{Permanent link}

http://nrs.harvard.edu/urn-3:HUL.InstRepos:9561259

\section{Terms of Use}

This article was downloaded from Harvard University's DASH repository, and is made available under the terms and conditions applicable to Open Access Policy Articles, as set forth at http:// nrs.harvard.edu/urn-3:HUL.InstRepos:dash.current.terms-of-use\#OAP

\section{Share Your Story}

The Harvard community has made this article openly available.

Please share how this access benefits you. Submit a story.

Accessibility 


\title{
Belief Disagreements and Collateral Constraints
}

\author{
Alp Simsek*
}

July 29,2012

\begin{abstract}
Belief disagreements have been suggested as a major contributing factor to the recent subprime mortgage crisis. This paper theoretically evaluates this hypothesis. I assume that optimists have limited wealth and take on leverage in order to take positions in line with their beliefs. To have a significant effect on asset prices, they need to borrow from traders with pessimistic beliefs using loans collateralized by the asset itself. Since pessimists do not value the collateral as much as optimists do, they are reluctant to lend, which provides an endogenous constraint on optimists' ability to borrow and to influence asset prices. I demonstrate that the tightness of this constraint depends on the nature of belief disagreements. Optimism concerning the probability of downside states has no or little effect on asset prices because these types of optimism are disciplined by this constraint. Instead, optimism concerning the relative probability of upside states could have significant effects on asset prices. This asymmetric disciplining effect is robust to allowing for short selling because pessimists that borrow the asset face a similar endogenous constraint. These results emphasize that what investors disagree about matters for asset prices, to a greater extent than the level of disagreements. When richer contracts are available, relatively complex contracts that resemble some of the recent financial innovations in the mortgage market endogenously emerge to facilitate betting.
\end{abstract}

JEL Codes: G1, G12, E3, D53

Keywords: Belief disagreements, heterogoeneous priors, collateral constraints, leverage, margin, asymmetric disciplining, short selling.

*Harvard University (e-mail: asimsek@fas.harvard.edu). Earlier versions of this paper have been circulated under the title, "When Optimists Need Credit: Asymmetric Disciplining of Optimism and Implications for Asset Prices." I am grateful to my advisor, Daron Acemoglu, for invaluable guidance and numerous suggestions that significantly improved the paper. I thank Aytek Erdil, Sam Kruger, Muhamet Yildiz, three anonymous referees, and the editor, Stephen Morris, for numerous helpful comments. I also thank Marios Angeletos, Roland Benabou, Ricardo Caballero, Melissa Dell, Emmanuel Farhi, Guido Lorenzoni, Ivan Werning and the seminar participants at the University of California-Berkeley, Brown University, the University of Chicago, Chicago Fed, Columbia University, Duke University, the European University Institute, Harvard University, IMF, MIT, New York University, Northwestern University, the University of Pennsylvania, Princeton University, Stanford University, Toulouse University, the University of Warwick, Yale University for helpful comments. All remaining errors are mine. 


\section{Introduction}

The recent subprime mortgage crisis highlighted the importance of investors' belief disagreements for asset prices. A number of commentators, e.g., Shiller (2005) and Reinhart and Rogoff (2008), have identified the optimism of a fraction of investors as a potential cause for the increase in asset prices in the run-up to the crisis in the markets for housing and mortgage backed securities. The optimistic buyers in these markets have often financed their asset purchases by borrowing from other, potentially less optimistic, investors. Much of this borrowing, e.g., mortgages, margin loans, or REPO loans, has been collateralized by the asset itself. In these transactions, the fraction of the asset price buyers pay is referred to as the margin. Geanakoplos (2010) reports that the average margin used to purchase $\$ 2.5$ trillion worth of so-called toxic mortgage backed securities was about $6 \%$ in 2006, while it dramatically increased to over $80 \%$ in 2009 . Why was it easy for optimists to borrow before the crisis? Why was it difficult during the crisis?

To address these questions, this paper presents a model of credit markets with belief disagreements. Two types of traders with heterogeneous prior beliefs (optimists and pessimists) invest in a risky asset or (riskless) cash. Traders can also borrow cash or the asset from each other. The model features a standard collateral constraint: All borrowing contracts must be secured by collateral (cash or the asset) which the borrower owns. If the borrower's promised payment exceeds the value of collateral, then she defaults on the contract and the lender receives the collateral. Different borrowing contracts are available for trade at competitive prices. The contracts that are actually traded, along with the price of the asset, are determined in general equilibrium.

The model reveals an endogenous borrowing constraint which has the potential to shed some light on borrowing contracts observed before and during the crisis. The constraint stems from borrowers' and lenders' belief disagreements. To fix ideas, consider optimists that purchase the asset by borrowing cash from pessimists. Pessimists might be reluctant to lend because they do not value optimists' collateral (the asset) as much as optimists do. This represents an endogenous constraint on optimists' ability to borrow and to influence asset prices. A symmetric argument shows that belief disagreements also constrain pessimists' ability to borrow the asset from optimists. In this paper, I systematically analyze the implications of this constraint. In my baseline setting, borrowing the asset (short selling) is not allowed and borrowing cash is restricted to simple debt contracts that promise a fixed payment independent of the future state. These assumptions are useful to isolate optimists' borrowing constraints. I gradually relax the assumptions first by allowing for simple short contracts, which are useful to analyze pessimists' borrowing constraints; and then by considering richer contracts, which are useful to analyze the effect of financial innovation on borrowing constraints.

My main result, formalized in Theorems 2,4 concerns the baseline setting and shows that optimists face different endogenous constraints depending on the type of their optimism. To clarify, consider a simple example in which a single risky asset is traded. There are three states, good, normal and bad, in which the asset's future price will respectively be high, average and low. 
Pessimists assign an equal probability, $1 / 3$, to each state. In contrast, optimists have a greater expected valuation of the asset. In this example, one can imagine two different types of optimism. For the first case, suppose optimists assign a probability less than $1 / 3$ to the bad state, and equal probabilities to the normal and the good states. That is, optimism is on the downside in the sense that optimists think bad states are unlikely. Downside optimism might have applied during the recent financial crisis (in the Fall of 2008 and early 2009), when a main dimension of uncertainty was about the length and the scope of the economic recession 11 In this case, I show that optimists borrow by using loans with relatively high margins and the current price is relatively low (in particular, relatively close to pessimists' valuation). For the second case, suppose optimists agree with pessimists about the probability of the bad state, but they assign a greater probability to the good state than the normal state. That is, optimism is on the upside in the sense that optimists think good events are likely. Upside optimism might have applied in the run-up to the crisis, when a fraction of homebuyers thought house prices would continue to increase at a high rate (see Case and Shiller, 2003). One can construct this case such that optimists' valuation of the asset is the same as in the first case, so that optimism differs in type but not level. In this case, I show that optimists borrow by using loans with lower margins and the price is higher (in particular, closer to optimists' valuation).

One way to summarize this result is to note that optimism is asymmetrically disciplined by the endogenous borrowing constraint: Downside optimism is disciplined while upside optimism is not. The intuition for asymmetric disciplining is related to the asymmetry in the shape of the payoffs of collateralized loans. Optimists pay the loan in full in upside states, but they default in downside states. Consequently, optimism about the probability of downside states translates into a disagreement about how to value the loans, which tightens the endogenous borrowing constraint. This induces optimists to borrow using loans with higher margins on which there is relatively less disagreement. This in turn decreases optimists' demand for the asset and leads to a lower equilibrium price. In contrast, optimism about the relatively likelihood of upside states does not tighten the endogenous borrowing constraint. Optimists borrow with loans with lower margins and the asset price is higher.

The asymmetric disciplining result lends itself to a number of comparative statics results regarding the effect of belief disagreements on asset prices. Miller (1977) has shown that, with short selling constraints, an increase in belief disagreements tends to increase the overvaluation of the asset (relative to the average valuation in the population) because the asset is held by the most optimistic investors. In contrast, the level of belief disagreements in my baseline setting has ambiguous effects. This is because, while an increase in buyers' optimism tends to increase the price, an increase in lenders' pessimism tends to decrease the price through the tightening of the endogenous borrowing constraint. Theorem 5 qualifies the Miller mechanism for environments in which optimists finance their asset purchases by borrowing from less optimistic lenders. In

\footnotetext{
${ }^{1}$ The downside nature of the uncertainty during this period was also noted by Chairman Bernanke in his semiannual report to the Congress on February 24, 2009, when he said: "This outlook for economic activity is subject to considerable uncertainty, and I believe that, overall, the downside risks probably outweigh those on the upside."
} 
particular, an increase in belief disagreements increases the asset price (and decreases margins) if the additional disagreements concern non-default states, but it has the opposite effect if the disagreements are about default states. Put differently, what investors disagree about matters for asset prices, to a greater extent than the level of their disagreements.

A shortcoming of the Miller (1977) mechanism is its reliance on exogenous constraints on short selling, which restricts its applicability. In contrast, my asymmetric disciplining result continues to apply even if the asset can be short sold. I analyze short selling in an extension of the baseline setting with simple short contracts. Pessimists use these contracts to borrow the asset from optimists, which they then sell in the market. Pessimists use their cash holdings to collateralize their promise to return the asset. The key insight is that short contracts, just like debt contracts, default if the value of their promise (the asset) exceeds the value of the cash-collateral. This makes optimists reluctant to lend the asset, providing an endogenous constraint on pessimists' ability to short sell. I show that pessimism is also asymmetrically disciplined by this constraint. Moreover, since short contracts default in the symmetric-opposite states as debt contracts (high asset payoff vs. low asset payoff), the asymmetric disciplining of pessimism is complementary to the asymmetric disciplining of optimism. When belief disagreements are on the upside, the endogenous borrowing constraint is loose for optimists but tight for pessimists, which leads to a price closer to optimists' valuation. When the belief disagreements are on the downside, the situation is the opposite and the price is closer to pessimists' valuation. In particular, belief disagreements systematically affect asset prices despite the fact that optimists and pessimists can borrow with an "equally rich" set of contracts (i.e., simple debt and short contracts).

While simple debt and short contracts are common in financial markets, rapid financial innovation in recent years has made available richer contracts such as options, futures, and more complex derivatives. A natural question is how the availability of these contracts interact with optimists' or pessimists' borrowing constraints. I address these issues in an extension with unrestricted contracts, and obtain two main results. First, the asymmetric disciplining result is robust to the presence of richer contracts. Second, relatively complex contracts that resemble some of the recent innovations endogenously emerge to facilitate betting. In particular, the asset is tranched so that optimists receive the asset payoff only in sufficiently good states, while pessimists receive it in bad states. The tranching of the asset resembles the collateralized debt or mortgage obligations $(C D O s, C M O s)$ by which the payoff from the underlying asset is split across traders according to their different needs. Similarly, cash is also tranched so that pessimists receive a constant payoff in sufficiently bad states, while optimists receive the payoff in good states. The tranching of cash resembles credit default swaps (CDSs) or synthetic CDOs by which pessimists receive an insurance payment in bad states in exchange for paying insurance premiums in good states. Taken together, this analysis suggests that belief disagreements (or a related source of heterogeneity in valuations) might be a driving force behind some of the recent financial innovations.

Outline. The organization of the rest of this paper is as follows. The next subsection discusses the related literature. Section 2 describes the model and defines the equilibrium corresponding 
to a general set of borrowing contracts. Section 3 considers the baseline setting with simple debt contracts and characterizes the equilibrium. This section also presents the main result about the asymmetric disciplining of optimism. Section 4 analyzes the comparative statics of equilibrium with respect to the type and the level of belief disagreements. Sections 5 and 6 respectively present the extensions with simple short contracts and richer contracts. The paper ends with concluding remarks and several appendices that present omitted proofs and extensions.

\subsection{Related Literature}

My paper is closely related to the work of Geanakoplos (1997, 2003, 2010), who pioneered the general equilibrium analysis of collateralized lending and asset prices, and provided a plausible model of countercyclical loan margins. The distinctive feature of my analysis is the focus on the role of different types of belief disagreements.2 To capture this feature, I consider an extension of the Geanakoplos' (2003) model with a continuum of states (as opposed to two or three), which allows for a flexible specification of traders' beliefs. To keep the analysis tractable, I also consider only two belief types of traders, optimists and pessimists (as opposed to a continuum). My analysis offers a number of new insights. First, while Geanakoplos (2003) shows that an increase in belief disagreements can increase margins and decrease asset prices considerably, my paper qualifies this result and emphasizes the type of belief disagreements rather than the level. In the Geanakoplos' model, the increase in belief disagreements increases margins because the disagreements are concentrated on downside states. An increase in belief disagreements in that model would actually decrease margins (and increase asset prices) if the additional disagreements were about upside states. Second, I generalize the Geanakoplos' model by allowing for short selling which is not uncommon in financial markets. Third, I also generalize the model by allowing for unrestricted contracts. Geanakoplos (2010) shows that the introduction of the CDS contract reduces the asset price. My analysis complements this result by showing that relatively complex contracts some of which resemble the CDS endogenously emerge when the contract space is sufficiently rich. In recent work, Fostel and Geanakoplos (2012) analyze further the asset pricing implications for these contracts in the two state model.

Another distinctive feature of my model is that the loans traded in equilibrium are risky. Thus, my model is particularly suitable to study economic issues associated with default of collateralized loans, which were at the forefront during the recent subprime crisis. In contrast, the baseline setting in Geanakoplos $(2003,2010)$ features no default because loans are endogenously collateralized according to the worst case scenario. Fostel and Geanakoplos (2011) show that this is always the case when there are two continuation states and traders hold assets only because of the cash flows they generate. Put differently, to have default in equilibrium, one must consider either more than two continuation states as in this paper, or traders that receive non-pecuniary

\footnotetext{
${ }^{2}$ In recent work, Park and Sabourian (2010) show that the type of traders' beliefs also matters in a different context, learning in financial markets, to determine whether informational herding (following the crowd) or contrarianism (acting against the crowd) is observed.
} 
utility from owning the collateral (as could be the case for housing or cars). In related work, He and Xiong (2011) show that frequent trading further mitigates default and bankruptcy risk, because traders in equilibrium endogenously choose to borrow with shorter term debt.

My paper is part of a large theoretical literature that concerns the effect of borrowing constraints on asset prices, e.g., Shleifer and Vishny (1992, 1997), Kiyotaki and Moore (1997), Gromb and Vayanos (2002), Fostel and Geanakoplos (2008), Garleanu and Pedersen (2011), Brunnermeier and Pedersen (2009), Acharya, Gale and Yorulmazer (2009), Brunnermeier and Sannikov (2011) $!^{3}$ In addition, my results on loan margins are related to the corporate finance literature that concerns the determinants of leverage, e.g., Townsend (1979), Myers and Majluf (1984), Gale and Hellwig (1985), Hart and Moore (1994). The main difference from these literatures is the focus on belief disagreements as a friction that constrains borrowing, as opposed to asymmetric information, lack of commitment, or exogenously specified borrowing (or margin) constraints.

My results on short selling contribute to a literature that analyzes the frictions that constrain asset lending. Duffie (1996), D'Avolio (2001) and Lamont (2004) emphasize that the participation constraints of potential asset lenders (for legal, institutional, or behavioral reasons) are crucial to sustain positive short fees. Duffie, Garleanu and Pedersen (2002) analyze the role of search frictions in generating large short fees. My paper analyzes the role of collateral constraints in restricting asset lending, and shows that the short fees (and short margins) are also affected by the nature of traders' belief disagreements.

The relationship of my paper to the literature initiated by Miller (1977) has been discussed. In related work, Harrison and Kreps (1978) consider a dynamic asset pricing setting with belief disagreements in which the identity of optimists changes over time. When short selling is constrained, the asset price exceeds the present discounted valuation of any trader because of the resale option value to future optimists. As Scheinkman and Xiong (2003) note, this resale option value may be reasonably called a "speculative bubble." My results suggest that, when optimists are borrowing constrained, speculative bubbles financed by credit are more likely when belief disagreements concern upside states (see Simsek, 2010, for a formalization) $4^{4}$

\footnotetext{
${ }^{3}$ On the empirical side, recent work in asset pricing, e.g., Jurek and Stafford (2011), can explain the pricing of collateralized loans with different margins during the recent crisis, but cannot speak to the endogenous determination of margins. A growing literature documents balance sheet leverage and REPO margins during the crisis, e.g., Adrian and Shin (2010), Gorton and Metrick (2011), Copeland et al. (2010), Krishnamurthy et al. (2011).

${ }^{4}$ There is a large literature that analyzes the asset pricing implications of heterogeneous beliefs and short selling constraints. In addition to the mentioned papers, an incomplete list includes Jones and Lamont (2001), Chen, Hong and Stein (2002), Diether, Malloy and Scherbina (2002), Ofek and Richardson (2003), Lamont and Stein (2004). Papers that analyze speculative bubbles include Allen, Morris, and Postlewaite (1993), Morris (1996), Burnside, Eichenbaum and Rebelo (2011).

A related literature concerns the plausibility of heterogeneous prior beliefs in financial markets. Morris (1995) provides an excellent discussion of the merits of the common prior assumption, and argues that heterogeneous priors should be selectively incorporated into economic analysis. Another concern is whether belief disagreements would disappear in view of traders' Bayesian learning. Recent theoretical work (e.g., Acemoglu, Chernozhukov and Yildiz, 2009) has emphasized that Bayesian learning might fail to generate agreement even in the long run. A related concern is Friedman's (1953) market selection hypothesis, which posits that investors with incorrect beliefs should be driven out of the market as they would consistently lose money. Recent research (e.g., Sandroni, 2000, Blume and Easley, 2006, and Cao, 2011) has demonstrated the limitations of this hypothesis, by showing that traders with inaccurate (and heterogeneous) beliefs may have a permanent presence when financial markets are incomplete.
} 


\section{Basic Environment with Borrowing Constraints}

Consider an economy with two dates, denoted by $\{0,1\}$, and a single consumption good which will be referred to as a dollar. The economy has a continuum of risk neutral traders who have endowments at date 0 , but who only consume at date 1 . Traders can transfer their endowments to date 1 by investing in one of two ways. First, traders can keep their dollars in cash which yields one dollar at date 1 for each dollar invested at date 0. Cash is supplied elastically and its role is to fix the riskless interest rate for this economy, which is normalized to zero. Second, traders can also invest in a risky asset which is supplied inelastically at date 0 at a normalized supply of one unit. The asset yields dollars only at date 1 , and it is traded at date 0 at a price $p$ which will be endogenously determined.

There is a continuum of possible states at date 1 , denoted by $s \in \mathcal{S}=\left[s^{\min }, s^{\max }\right]$. The asset pays $s$ dollars if state $s$ is realized, so the state captures the uncertainty in the asset's payoff. Traders have heterogeneous priors about the state. In particular, there are two types of traders, optimists and pessimists, respectively denoted by subscript $i=1$ and $i=0$. Type $i$ traders' prior beliefs about the state is given by the probability distribution $F_{i}$ over $S$. Traders know each others' priors, that is, optimists and pessimists agree to disagree $5^{5}$ The probability distributions $F_{1}$ and $F_{0}$ have density functions $f_{1}, f_{0}$ which are continuous and positive over $\mathcal{S}$. Let $E_{i}[\cdot]$ denote the expectation operator corresponding to the belief of a type $i$ trader. Optimists are optimistic about the asset in the sense that:

$$
E_{1}[s]>E_{0}[s]
$$

In subsequent sections, this assumption will be strengthened by various regularity conditions.

Without loss of generality, I normalize the mass of each type of traders to 1 . Type $i$ traders are initially endowed with $n_{i}>0$ dollars and zero units of the asset. All units of the asset are initially endowed to unmodeled agents who sell their asset holdings at date 0 and consume. $!^{6}$ An economy is denoted by the tuple $\mathcal{E}=\left(\mathcal{S} ;\left\{F_{i}\right\} ;\left\{n_{i}\right\}\right)$.

In view of assumption (1), optimists are the natural buyers of the asset. In addition to investing their endowments, optimists might want to borrow cash from pessimists to increase their investments in the asset. Relatedly, pessimists might want to borrow the asset from optimists to short sell. The common feature in these arrangements is borrowing (either cash or the asset). I next describe the frictions that constrain borrowing in this economy.

\footnotetext{
${ }^{5}$ This assumption provides a simple model of trade based on belief disagreements. To the extent that prices do not fully reveal information, e.g., because of liquidity or noise traders, the present model could be viewed as a reduced form for a setting in which belief disagreements are driven by differences in information rather than priors. That said, models with information differences have some additional features, e.g., the possibility of market breakdowns, which are not captured by the present model.

${ }^{6}$ The only role of this assumption is to simplify the analysis by eliminating the feedback effect from asset prices to traders' net worths. See Simsek (2010) for an earlier version of the paper in which all assets are initially allocated to the traders in the economy. The results continue to apply as long as optimists initially do not hold the entire asset supply.
} 


\subsection{Borrowing Constraints in General Equilibrium}

All borrowing in this economy is subject to a collateral constraint. That is, promises made by borrowers must be collateralized by either the asset or the cash that they own. I model this constraint using a general equilibrium approach similar to Geanakoplos (2003, 2010). In this approach, borrowing contracts are not determined by a negotiation process between borrowers and lenders. Instead, contracts are treated as commodities that are traded in anonymous competitive markets. Traders choose their positions in all available contracts taking the prices of contracts as given. The contracts that are traded in non-zero quantities are determined in equilibrium. 7

Formally, a borrowing contract, $\beta \equiv\left([\boldsymbol{\varphi}(s)]_{s \in S}, \alpha, \gamma\right)$, is a promise of $\boldsymbol{\varphi}(s) \geq 0$ dollars in state $s$, collateralized by $\alpha \geq 0$ units of the asset and the $\gamma \geq 0$ units of cash. The contract is traded in an anonymous market at a competitive price $q(\beta)$ that will be endogenously determined. A trader who sells contract $\beta$, the borrower, borrows $q(\beta)$ dollars at date 0 . The borrower sets aside $\alpha$ units of the asset and $\gamma$ units of cash that she owns as collateral. A trader who buys this contract, the lender, lends $q(\beta)$ dollars at date 0 . The lender becomes entitled to a payment of $\boldsymbol{\varphi}(s)$ dollars in state $s$ of date 1 . However, the lender may not receive $\boldsymbol{\varphi}(s)$ dollars in full because the payment is only enforced by collateral. More specifically, if the future asset value $s$ is such that $\boldsymbol{\varphi}(s)>\alpha s+\gamma$, then the contract defaults and the lender only receives the value of the collateral, $\alpha s+\gamma$. Combining the default and the non-default events, the payoff of contract $\beta$ can be written as:

$$
\min (\alpha s+\gamma, \varphi(s))
$$

This framework can account for various different forms of collateralized borrowing arrangements, as illustrated by the following examples.

1. Simple debt contracts. Consider a contract that satisfies $\varphi(s) \equiv \varphi$ for some $\varphi \in \mathbb{R}_{+}$. This corresponds to a debt contract in which the borrower promises a fixed payment of $\varphi$ dollars at date 1 . The debt contract is simple in the sense that the borrower's payment does not depend on the future state. Simple debt contracts provide a model of some common collateralized loans (e.g., REPOs, mortgages, asset purchases on margin) which do not have many contingencies. Sections 3.3 and 4 (the baseline setting) analyze the equilibrium corresponding to this set of contracts.

2. Simple short contracts. Consider a contract that satisfies $\varphi(s) \equiv \varphi s$ for some $\varphi \in \mathbb{R}_{+}$. This corresponds to a short contract in which the borrower promises to return $\varphi$ units of the asset at date 1 . The short contract is simple in the sense that the promised number of assets does not depend on the future state. Simple short contracts provide a model of short selling the asset. Section 5 analyzes the equilibrium corresponding to this set of contracts.

\footnotetext{
${ }^{7}$ The general approach of treating contracts as commodities has been pioneered by Prescott and Townsend (1984a, 1984b). The equilibrium notion in this section has been first developed by Geanakoplos and Zame (1997, 2009).
} 
I define the contract space as:

$$
\mathcal{B}=\left\{(\varphi, \alpha, \gamma) \mid \begin{array}{c}
\varphi: \mathcal{S} \rightarrow \mathbb{R}_{+} \text {is measurable and bounded, } \\
\alpha \in \mathbb{R}_{+}, \text {and } \gamma \in \mathbb{R}_{+} .
\end{array}\right\}
$$

I equip $\mathcal{B}$ with the sup norm, which makes it into a Banach space. The rest of this section defines the general equilibrium corresponding to an exogenously specified subspace, $\mathcal{B}^{T} \subset \mathcal{B}$. The subsequent sections characterize this equilibrium for different specifications for $\mathcal{B}^{T}$.

Let $\mathcal{P}\left(\mathcal{B}^{T}\right)$ denote the space of finite Borel measures over $\mathcal{B}^{T}$, endowed with the weak ${ }^{*}$ topology. I model traders' contract choices as elements in $\mathcal{P}\left(\mathcal{B}^{T}\right)$. To keep the notation simple, I assume that all traders of the same type make identical choices (This assumption is innocuous as explained in Footnote 9). In particular, type $i$ traders choose two measures, $\mu_{i}^{+}, \mu_{i}^{-}$. The measure, $\mu_{i}^{+}$, represents traders' positive positions on contracts: that is, it captures the contracts through which type $i$ traders lend. The measure, $\mu_{i}^{-}$, represents traders' negative positions in contracts: that is, it captures the contracts through which type $i$ traders borrow. In addition, I assume that the price function, $q: \mathcal{B}^{T} \rightarrow \mathbb{R}_{+}$is restricted to be Borel measurable and bounded, which also makes it integrable with respect to $\mu_{i}^{+}$and $\mu_{i}^{-}$. Then, type $i$ traders' budget constraint can be written as:

$$
p a_{i}+c_{i}+\int_{\beta \in \mathcal{B}^{T}} q(\beta) d \mu_{i}^{+}-\int_{\beta \in \mathcal{B}^{T}} q(\beta) d \mu_{i}^{-} \leq n_{i}
$$

Here, $a_{i} \in \mathbb{R}_{+}$denotes the traders' asset demand, $c_{i} \in \mathbb{R}_{+}$denotes their cash holdings, and the integral terms respectively capture their lending and borrowing. Note that the negative positions, $\mu_{i}^{-}$, enable traders to borrow and expand their budget. However, borrowing is subject to the collateral constraints:

$$
\begin{aligned}
& \int_{\left([\boldsymbol{\varphi}(s)]_{s \in S}, \alpha, \gamma\right) \in \mathcal{B}^{T}} \alpha d \mu_{i}^{-} \leq a_{i}, \text { and } \\
& \int_{\left([\boldsymbol{\varphi}(s)]_{s \in S}, \alpha, \gamma\right) \in \mathcal{B}^{T}} \gamma d \mu_{i}^{-} \leq c_{i} .
\end{aligned}
$$

In particular, traders must own sufficiently many assets and cash to pledge as collateral for the contracts they sell. Note that there is no analogous condition for traders' positive positions, $\mu_{i}^{+}$, because lending does not require collateral.

Type $i$ traders choose their positions to solve:

$$
\max _{\left(a_{i}, c_{i}\right) \in \mathbb{R}_{+}^{2} ; \mu_{i}^{+}, \mu_{i}^{-}} \quad a_{i} E_{i}[s]+c_{i}+E_{i}\left[\int_{\mathcal{B}^{T}} \min (\boldsymbol{\varphi}(s), \alpha s+\gamma) d \mu_{i}^{+}\right],
$$

subject to (3) and (4). 
Note that traders calculate their expected payoffs (and payments) on assets and contract portfolios according to their own beliefs. The market for contracts is competitive. In particular, the price function $q(\cdot)$ is determined by debt market clearing. Since there is a mass 1 of each type of traders, the market clearing condition can be written as:

$$
\mu_{1}^{+}+\mu_{0}^{+}=\mu_{1}^{-}+\mu_{0}^{-}
$$

Definition 1. A general equilibrium is a collection of prices $\left(p \in \mathbb{R}_{+}, q: \mathcal{B}^{T} \rightarrow \mathbb{R}_{+}\right)$and portfolios $\left(\hat{a}_{i}, \hat{c}_{i}, \hat{\mu}_{i}^{+}, \hat{\mu}_{i}^{-}\right)_{i \in\{0,1\}}$ such that the portfolios solve Problem (5) for each $i \in\{0,1\}$, the asset market clears, $\sum_{i \in\{0,1\}} \hat{a}_{i}=1$, and debt markets clear [cf. Eq. (6)].

\section{Equilibrium with Simple Debt Contracts}

This section characterizes the general equilibrium in the baseline setting with simple debt contracts. It also presents the main result which shows that optimism is asymmetrically disciplined by borrowing constraints.

Recall that a simple debt contract is denoted by $\left([\boldsymbol{\varphi}(s)=\varphi]_{s \in S}, \alpha, \gamma\right)$ for some $\varphi \in \mathbb{R}_{+}$. In addition, I assume the cash-collateral is zero, $\gamma=0$, which is without loss of generality in this section 8 I also normalize the contracts by taking the asset-collateral to be one, i.e., $\alpha=1$. Under these assumptions (and normalization), the set of traded contracts is given by:

$$
\mathcal{B}^{D} \equiv\left\{\left([\varphi(s) \equiv \varphi]_{s \in S}, 1,0\right) \mid \varphi \in \mathbb{R}_{+}\right\}
$$

When there is no confusion, I denote a simple debt contract in $\mathcal{B}^{D}$ by its promised payment, $\varphi$.

Restricting attention to the contract set $\mathcal{B}^{D}$ represents two frictions in addition to the collateral constraint. The first friction is the absence of short selling. The second friction is the absence of more general debt contracts which may promise payments contingent on the asset's value $s$. It is natural to start with simple debt contracts, and defer the analysis of richer contracts to later sections, for a number of reasons. First, many assets other than stocks are difficult and costly to short sell, and many common collateralized loan arrangements (e.g., REPOs or asset purchases on margin) do not feature contingencies. Second, considering simple debt contracts conforms well with a strand of the financial frictions literature that focuses on simple and riskless debt contracts (e.g., Kiyotaki and Moore, 1997, or more recently, Brunnermeier and Sannikov, 2011). The analysis in this section is more general than this strand of the literature because optimists are allowed to borrow with contracts that might default in some future states.

To characterize the equilibrium, I first consider an alternative principal-agent model of borrowing constraints which is more tractable than the general equilibrium model. I proceed by establishing the equivalence of the equilibria of the two models. I then characterize the principal-

\footnotetext{
${ }^{8}$ To see this, consider a contract $(\varphi, \alpha, \gamma)$ with $\gamma>0$. The transfers generated by this contract can be equivalently captured by the contract, $(\max (0, \varphi-\gamma), \alpha, 0)$.
} 
agent equilibrium. The equivalence result not only provides a method of solving the general equilibrium, but it also clarifies (in the context of economy $\mathcal{E}$ ) the relationship between the general equilibrium and the principal-agent models of borrowing constraints.

\subsection{An Alternative Principal-Agent Model of Borrowing Constraints}

As an alternative to the general equilibrium model, one could imagine that contracts are determined as the result of a contractual negotiation process between borrowers and lenders. In this case, it would be possible to characterize the set of constrained efficient contracts. However, the contract allocation within this set would depend on the allocation of bargaining power between traders (as well as the details of the bargaining process). To make progress, it is common in the literature to focus on the special case in which borrowers have all the bargaining power (cf., Gale and Hellwig, 1985 and Holmstrom and Tirole, 1997). This leads to a principal-agent approach: The borrower chooses the debt contract subject to a set of frictions and lenders' participation constraint.

I next consider this approach for the economy $\mathcal{E}$. Suppose optimists (who are the natural borrowers with simple debt contracts) choose their borrowing and outstanding debt subject to a participation constraint for pessimists (who are the natural lenders). To eliminate corner cases, I also make the following assumption:

Assumption (A1). $n_{1}<E_{1}[s]-s^{\min }$ and $n_{0}>E_{1}[s]-n_{1}$.

The first part of the assumption ensures that optimists (in equilibrium) cannot fund their asset purchases with riskless debt. The second part ensures that pessimists' endowment is sufficiently large to meet optimists' borrowing demand. Given assumption (A1), I will establish that the equilibrium price satisfies $p \in\left(E_{0}[s], E_{1}[s]\right)$. Since the price is greater than the pessimistic valuation, pessimists have no interest in investing in the asset. They use their endowment to invest in cash and to lend to optimists. In contrast, optimists make leveraged investments in the asset. The remaining question is how much optimists borrow and how many assets they demand.

To address this question, let $a_{1}$ denote optimists' asset position and $\varphi$ denote their outstanding debt per-asset at date 1 . In view of the collateral constraint, optimists' actual payment on their debt is given by $\min (s, \varphi)$. Consequently, lenders' participation constraint implies that their lending per-asset at date 0 is given by $E_{0}[\min (s, \varphi)]$. Optimists choose their asset position and outstanding debt to solve:

$$
\begin{aligned}
\max _{\left(a_{1}, \varphi\right) \in \mathbb{R}_{+}^{2}} & a_{1} E_{1}[s]-a_{1} E_{1}[\min (s, \varphi)], \\
\text { s.t. } & a_{1} p=n_{1}+a_{1} E_{0}[\min (s, \varphi)] .
\end{aligned}
$$

The first line is optimists' expected payoff at date 1: They receive a payoff from the asset but they make a payment on their debt. The second line is optimists' budget constraint which incorporates lenders' participation constraint. 
Definition 2. Given assumption (A1), a principal-agent equilibrium is a pair of asset price $p$ and optimists' portfolio $\left(a_{1}^{*}, \varphi^{*}\right)$, such that optimists' portfolio solves problem (8) and the asset market clears, that is, $a_{1}^{*}=1$.

The principal-agent equilibrium will be characterized in Section 3.3. The characterization (and the equivalence result in Section 3.2 requires the following regularity condition on beliefs, which ensures that problem (8) has a unique solution for each $p$.

Assumption (A2). The probability distributions $F_{1}$ and $F_{0}$ satisfy the hazard-rate order:

$$
\frac{f_{1}(s)}{1-F_{1}(s)}<\frac{f_{0}(s)}{1-F_{0}(s)} \text { for each } s \in\left(s^{\min }, s^{\max }\right)
$$

The hazard-rate order is equivalent to saying that $\frac{1-F_{1}(s)}{1-F_{0}(s)}$ is strictly increasing over $\mathcal{S}$. Intuitively, this notion of optimism concerns optimists' relative probability assessment for the upperthreshold events $\left[s, s^{\max }\right] \subset \mathcal{S}$. It posits that optimists are increasingly optimistic for these events as the threshold level $s$ is increased. It captures the idea that, the "better" the event becomes, the greater the optimism is for the event. The hazard-rate order is related to some well known regularity conditions. It is stronger than the first order stochastic order, that is, the inequality in (9) implies that $F_{1}$ dominates $F_{0}$ in the first order stochastic sense. However, it is weaker than the monotone likelihood ratio property: that is, if $\frac{f_{1}(s)}{f_{0}(s)}$ is strictly increasing over $\mathcal{S}$, then the inequality in 9 holds.

\subsection{Equivalence of the Principal-Agent and the General Equilibrium}

The principal-agent approach is useful for its simplicity and tractability. However, the assumption that optimists have the bargaining power requires motivation. In contrast, the general equilibrium approach does not a priori take a stance on traders' bargaining powers. It is perhaps fortunate that for this economy the two approaches are equivalent, as shown by the following result.

Theorem 1 (Existence, Uniqueness, and Equivalence of Equilibria). Suppose the contract space is restricted to simple debt contracts, $\mathcal{B}^{T}=\mathcal{B}^{D}$, and assumptions (A1) and (A2) hold.

(i) There exists a unique principal-agent equilibrium $\left[p^{*},\left(a_{1}^{*}, \varphi^{*}\right)\right]$ with $p^{*} \in\left(E_{0}[s], E_{1}[s]\right)$.

(ii) There exists a general equilibrium $\left[(\hat{p},[q(\cdot)]),\left(\hat{a}_{i}, \hat{c}_{i}, \hat{\mu}_{i}^{+}, \hat{\mu}_{i}^{-}\right)_{i \in\{0,1\}}\right]$. In this equilibrium, optimists borrow and pessimists lend, i.e., $\hat{\mu}_{1}^{+}=\hat{\mu}_{0}^{-}=0$. Moreover, only a single contract is traded in non-zero quantities, that is, $\hat{\mu}_{1}^{-}$(and thus, $\hat{\mu}_{0}^{+}$) is a Dirac measure that puts weight only at one contract $\hat{\varphi} \in \mathcal{B}^{D}$. The general equilibrium is essentially unique in the sense that the portfolios, the asset price, $\hat{p}$, and the price of the traded debt contract, $q(\hat{\varphi})$, are uniquely determined.

(iii) The general equilibrium and the principal-agent equilibrium are equivalent:

$$
\hat{p}=p^{*}, \hat{a}_{1}=a_{1}^{*}=1, \hat{\varphi}=\varphi^{*} \text {, and } q(\hat{\varphi})=E_{0}\left[\min \left(s, \varphi^{*}\right)\right]
$$

The first part establishes the existence and the uniqueness of the principal-agent equilibrium. The second part establishes the existence and the essential uniqueness of the general equilibrium. 
The only source of non-uniqueness is the price of non-traded debt contracts, $q(\varphi)$ for $\varphi \neq \hat{\varphi}$. However, this non-uniqueness is inessential in the sense that it does not affect the equilibrium portfolios or prices of traded contracts. 9 The third part of the theorem establishes that the general equilibrium is identical to the principal-agent equilibrium. More specifically, in either equilibrium optimists have the same outstanding debt per-asset, $\hat{\varphi}=\varphi^{*}$, and they borrow the same amount, $q(\hat{\varphi})=E_{0}\left[\min \left(s, \varphi^{*}\right)\right]$, which also leads to the same asset price, $\hat{p}=p^{*}$.

Put differently, the general equilibrium approach results in contracts as if optimists have all the bargaining power. There is a simple explanation for this result. Since traders' endowments are assumed to be sufficiently large, $n_{0}+n_{1}>E_{1}[s]$, some traders must hold cash in general equilibrium. Since cash yields a gross return of 1 and it is not used as collateral, those traders must receive a gross return of 1 on their net worth. Since the price is interior, $p^{*} \in\left(E_{0}[s], E_{1}[s]\right)$, optimists can always secure a gross return higher than 1 by simply holding the asset. In contrast, pessimists cannot receive a higher return in the asset market since short selling is not allowed. Thus, the traders that hold cash in equilibrium must be the pessimists. It follows that pessimists' participation constraint binds and optimists receive all of the surplus from trade.

Intuitively, when the exogenously specified contract set includes only simple debt contracts, the competitive equilibrium favors optimists. With simple debt contracts, the asset is useful as collateral while cash is not. Thus, pessimists, who are the natural holders of cash, compete to make loans to optimists, rather than the opposite. In line with this intuition, Section 5 characterizes the polar opposite case with only simple short contracts and shows that the general equilibrium results in allocations as if pessimists have all the bargaining power.

\subsection{Optimists' Optimal Contract}

I next turn to the characterization of the principal-agent equilibrium. The next result, which is the main result, characterizes optimists' contract choice for a given price $p$. The next subsection combines this analysis with asset market clearing to solve for the equilibrium asset price.

Recall that optimists' outstanding debt per-asset is denoted by $\varphi$. Thus, optimists default on their debt if and only if the future asset value, $s$, is lower than the threshold state, $\bar{s} \equiv \varphi$. Consequently, I will refer to this simple debt contract as a loan with riskiness $\bar{s}$. Recall also that optimists' borrowing per-asset is given by $E_{0}[\min (s, \bar{s})]$, which I refer to as the size of the loan. The size of the loan is increasing in its riskiness: that is, larger loans are also riskier loans. Given these definitions, one interpretation of problem (8) is that optimists choose from a menu of loans with different sizes (and thus, riskiness levels) which are priced by pessimists. The next result

\footnotetext{
${ }^{9}$ To keep the notation simple, I have formulated the model so that all traders of a given type (i.e., all optimists and all pessimists) choose identical portfolios. Thus, Theorem 1 establishes the essential uniqueness of the general equilibrium within this symmetric class. However, the equilibrium is in fact essentially unique also within the larger class of allocations in which traders of a given type are allowed to behave differently. All optimists endogenously choose the same portfolio because, in equilibrium, there is a unique solution to their optimization problem (8) (see the proof in Appendix A.2. Pessimists do not necessarily choose the same portfolios since they are indifferent between lending to optimists and investing in cash. However, this is an inessential form of uniqueness which does not affect traders' returns or the asset price in equilibrium.
} 
characterizes the optimal loan.

Theorem 2 (Asymmetric Disciplining of Optimism). Suppose assumptions (A1) and (A2) hold, fix asset price $p$ that satisfies $p \in\left(E_{0}[s], E_{1}[s]\right)$, and consider optimists' problem (8). The riskiness, $\bar{s}$, of the optimal loan is the unique solution to the following equation over $\mathcal{S}$ :

$$
p=p^{o p t}(\bar{s}) \equiv \int_{s^{\min }}^{\bar{s}} s d F_{0}+\left(1-F_{0}(\bar{s})\right) \int_{\bar{s}}^{s^{\max }} s \frac{d F_{1}}{1-F_{1}(\bar{s})} .
$$

I will shortly provide a sketch proof of this result along with an intuition. Before doing so, it is useful to note a few important aspects of the function, $p^{\text {opt }}(\cdot)$. First, this function is similar to an inverse demand function: Given the price on the y-axis, it describes the riskiness of the optimal loan on the x-axis. Second, assumption (A1) implies $p^{\text {opt }}(\cdot)$ is strictly decreasing and continuous (cf. Appendix A.1). Since $p^{\text {opt }}\left(s^{\min }\right)=E_{1}[s]$ and $p^{o p t}\left(s^{\max }\right)=E_{0}[s]$, this further implies that there is a unique solution to Eq. (11).

Note also that $p^{\text {opt }}(\cdot)$ describes the equilibrium asset price conditional on the equilibrium loan riskiness $\bar{s}$. In particular, rewriting Eq. (11) yields the following asset pricing formula:

$$
p^{o p t}(\bar{s})=F_{0}(\bar{s}) E_{0}[s \mid s<\bar{s}]+\left(1-F_{0}(\bar{s})\right) E_{1}[s \mid s \geq \bar{s}] .
$$

This formula shows that optimism is asymmetrically disciplined in equilibrium. More specifically, the asset is priced with a mixture of optimistic and pessimistic beliefs. Pessimistic beliefs are used to assess the probability of default, $F_{0}(\bar{s})$, as well as the value of the asset conditional on default, $E_{0}[s \mid s<\bar{s}]$, while the optimistic beliefs are used to assess the value of the asset conditional on no default, $E_{1}[s \mid s \geq \bar{s}]$. Consequently, optimism about the probability of default states will not affect the asset price, while optimism about the relative probability of non-default states will increase the price. The following example further illustrates this asymmetric disciplining property.

Example 1 (Asymmetric Disciplining of Optimism). Consider the state space $\mathcal{S}=[1 / 2,3 / 2]$. Consider the following two cases that differ in the type of optimism.

Case (i). First suppose pessimists and optimists have the prior belief distributions $F_{0}$ and $F_{1, D}$ with density functions:

$$
f_{0}(s)=1 \text { for each } s \in \mathcal{S}, \quad f_{1, D}(s)=\left\{\begin{array}{ccc}
0.4 & \text { if } s \in \mathcal{S}_{B} \equiv[2 / 3-1 / 6,2 / 3+1 / 6) \\
1.3 & \text { if } s \in \mathcal{S}_{N} \equiv[1-1 / 6,1+1 / 6) \\
1.3 & \text { if } s \in \mathcal{S}_{G} \equiv[4 / 3-1 / 6,4 / 3+1 / 6]
\end{array} .\right.
$$

Here, $\mathcal{S}_{B}, \mathcal{S}_{N}$, and $\mathcal{S}_{G}$ capture "bad", "normal" and "good" events. Pessimists find all states equally likely while optimists have downside optimism in the sense that they think a bad event is unlikely 10

\footnotetext{
${ }^{10}$ Note that the belief distributions have discontinuous densities and they satisfy the hazard rate inequality, (9), only weakly. These distributions are used for illustration purposes because they provide a clear intuition. For analytical tractability, the formal results assume that beliefs have continuious densities and they satisfy the
} 

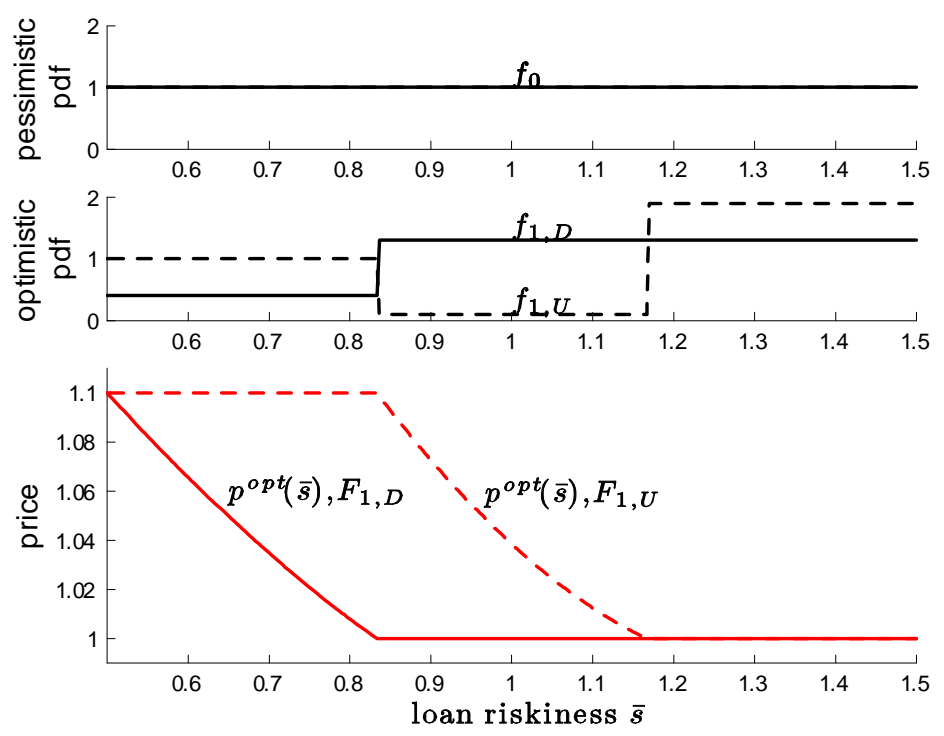

Figure 1: The top two panels display the probability density functions for traders' beliefs in the two scenarios of Example 1. The bottom panel displays the corresponding optimality curves, $p^{o p t}(\bar{s})$, the inverse of which gives the optimal loan riskiness $\bar{s}$ for a given price level $p$.

Case (ii). Next suppose pessimists have the same belief, but optimists' belief is changed to the distribution $F_{1, U}$ with density function

$$
f_{1, U}=\left\{\begin{array}{cl}
1 & \text { if } s \in \mathcal{S}_{B} \\
0.1 & \text { if } s \in \mathcal{S}_{N} \\
1.9 & \text { if } s \in \mathcal{S}_{G}
\end{array}\right.
$$

That is, optimists have upside optimism in the sense that they think a good event is more likely than a normal event (while they agree with pessimists about the probability of the bad event). Note also that optimists are equally optimistic in both cases, that is, $E_{1, U}[s]=E_{1, D}[s]$.

The bottom panel of Figure 1 displays the optimality curves, $p^{\text {opt }}(\cdot)$, corresponding to the two cases. For any price p, optimists choose a larger and riskier loan in the second case (with upside optimism) than the first case (with downside optimism). Equivalently, for any level of loan riskiness $\bar{s}$, the asset price is higher in the second case than in the first case, illustrating the asymmetric disciplining of optimism.

I next present a sketch proof of Theorem 2, which will be useful to provide the intuition. Optimists' problem (8), after substituting $a_{1}$ from the budget constraint, can be written as maximizing $n_{1} R_{1}^{L}(\bar{s})$, where:

inequality in $(9)$ strictly. 


$$
R_{1}^{L}(\bar{s}) \equiv \frac{E_{1}[s]-E_{1}[\min (s, \bar{s})]}{p-E_{0}[\min (s, \bar{s})]} .
$$

This expression is the return of optimists who buy one unit of the asset and who finance part of the purchase using a loan with riskiness $\bar{s}$. The denominator is the downpayment optimists make: they borrow $E_{0}[\min (s, \bar{s})]$ from pessimists and they pay the rest of the purchase. The numerator is optimists' expected payoff: they expect to receive $E_{1}[s]$ from the asset and they also expect to pay $E_{1}[\min (s, \bar{s})]$ on their loan. Appendix A.1 shows that $R_{1}^{L}(\bar{s})$ has a unique maximum characterized by the first order condition. The first order condition is given by $p=p^{\text {opt }}(\bar{s})$, which completes the sketch proof of Theorem 4

For intuition, it is useful to break down $R_{1}^{L}(\bar{s})$ into two components. First consider the left hand side terms in the numerator and the denominator of $(13)$, which constitute the unleveraged return:

$$
R_{1}^{U}=\frac{E_{1}[s]}{p} .
$$

This expression is the expected return of optimists if they buy the asset without borrowing. Since the theorem concerns prices that satisfy $p<E_{1}[s]$, the unleveraged return satisfies $R_{1}^{U}>1$. That is, optimists perceive that investing in the asset yields a higher return than investing in the storage technology. This creates a force that pushes optimists towards taking larger and riskier loans to invest more in the asset.

However, there is a second force that operates in the opposite direction. This force is related to the right hand side terms in the numerator and the denominator of (13), which constitute optimists' perceived interest rate on the loan:

$$
1+r_{1}^{p e r}(\bar{s}) \equiv \frac{E_{1}[\min (s, \bar{s})]}{E_{0}[\min (s, \bar{s})]}
$$

Optimists borrow $E_{0}[\min (s, \bar{s})]$ on the loan, but they expect to pay $E_{1}[\min (s, \bar{s})]$, which leads to the perceived interest rate $r_{1}^{\text {per }}(\bar{s})$. Assumption (A1) implies that $r_{1}^{\text {per }}(\bar{s})$ is always weakly greater than the riskless rate of 0 , and that it is increasing in $\bar{s}$ [cf. Appendix A.1]. This in turn creates a force that pushes optimists towards taking smaller and safer loans. The intuition for the properties of $r_{1}^{\text {per }}(\cdot)$ relies on two observations. First, collateralized loans always trade at an interest rate with a spread over the riskless rate because lenders require compensation for their expected losses in case of default. Moreover, since the loan market is competitive, the spread on a loan is just enough to compensate lenders according to their pessimistic belief. Second, optimists believe that the loan will default less often than pessimists do. Hence optimists think they will end up paying the full loan amount more often. Consequently, optimists perceive that they will pay a greater interest rate than the riskless rate. Moreover, for greater levels of $\bar{s}$, the scope of disagreement for default is greater, which implies that $r_{1}^{\text {per }}(\bar{s})$ is increasing in $\bar{s}$.

It follows that, while a larger and riskier loan enables optimists to take larger positions on the asset, it also comes at a greater perceived interest rate, $r_{1}^{\text {per }}(\bar{s})$. Optimists' optimal loan choice 
balances these two forces. This breakdown of forces also provides an intuition for the observation that the optimality curve, $p^{\text {opt }}(\bar{s})$, is decreasing. When the price is lower, optimists' unleveraged return, $R_{1}^{U}=\frac{E_{1}[s]}{p}$, is greater. This induces optimists to borrow more by taking a larger and riskier loan, agreeing to pay a greater perceived interest rate at the margin.

To see the intuition for the asymmetric disciplining, fix a loan with riskiness $\bar{s}$, and consider how much the price should drop (from the optimistic valuation) to entice optimists to take this particular loan. Consider this question in the context of Example 1 for a riskiness level $\bar{s}=0.8 \in$ $\mathcal{S}_{B}$. In the first case of Example 1, optimists find the bad event $\mathcal{S}_{B}$ unlikely. Hence, given a loan with riskiness $\bar{s} \in \mathcal{S}_{B}$, there is disagreement about the probability of default, which implies $r_{1}^{\text {per }}(\bar{s})>0$. As this loan appears expensive to optimists, the asset price should drop considerably to entice optimists to take this loan. Consider instead the second case of Example 1. In this case, for a loan with riskiness $\bar{s} \in S_{B}$, there is no disagreement about the probability of default, which implies $r_{1}^{\text {per }}(\bar{s})=0$. As the loan appears cheap to optimists, the asset price does not need to fall to entice them to take the loan (see Figure 1).

In other words, the asymmetric disciplining result operates through optimists' borrowing constraints. When the optimism is on the downside, optimists perceive tighter borrowing constraints [captured by a higher $r_{1}^{\text {per }}(\bar{s})$ ], which lowers their demand and leads to an asset price closer to pessimists' valuation. In contrast, upside optimism generates looser borrowing constraints and leads to an asset price closer to optimists' valuation.

\subsection{Asset Market Clearing}

I next consider asset market clearing and solve for the equilibrium. The budget constraint of problem (8) characterizes optimists' asset demand as:

$$
a_{1}=\frac{n_{1}}{p-E_{0}[\min (s, \bar{s})]}
$$

The denominator of this expression is the downpayment optimists make to buy one unit of asset, using a loan with riskiness $\bar{s}$ to finance the rest of the purchase. The numerator is their endowment, all of which they spend to purchase assets. Market clearing requires equating optimists' asset demand in 15 with the asset supply of 1 , which leads to:

$$
p=p^{m c}(\bar{s}) \equiv n_{1}+E_{0}[\min (s, \bar{s})] .
$$

Note that the market clearing curve, $p^{m c}(\bar{s})$, is increasing: When optimists take a larger and riskier loan, their demand for the asset is greater, which leads to a higher market clearing price. The equilibrium is then found as the unique intersection of the increasing market clearing curve, $p^{m c}(\bar{s})$, and the decreasing optimality curve, $p^{\text {opt }}(\bar{s})$. The following result summarizes the characterization of equilibrium.

Theorem 3. Suppose the contract space is restricted to simple debt contracts, $\mathcal{B}^{T}=\mathcal{B}^{D}$, and 
assumptions (A1) and (A2) hold. There is a unique principal agent equilibrium price and contract pair, $p^{*} \in\left(E_{0}[s], E_{1}[s]\right)$ and $\bar{s}^{*} \in S$, characterized as the solution to the following equations:

$$
p=p^{m c}(\bar{s})=p^{o p t}(\bar{s}),
$$

where $p^{m c}(\cdot)$ and $p^{\text {opt }}(\cdot)$ are respectively defined in Eqs. (11) and (16).

\section{The Type and the Level of Belief Disagreements}

This section establishes the comparative statics of equilibrium with respect to the type and the level of belief disagreements. In addition to the equilibrium loan riskiness, $\bar{s}^{*}$, and the asset price, $p$, an important variable is the margin on the loan:

$$
m \equiv \frac{p-E_{0}\left[\min \left(s, \bar{s}^{*}\right)\right]}{p} .
$$

That is, the margin is the fraction of the price optimists pay out of their own pocket, while financing the rest of the purchase by using the collateralized loan. The comparative statics of the margin are important for a couple reasons. First, margins are readily observable for some common collateralized lending arrangements (e.g., REPOs, mortgages, or margin purchases). Second, recent studies, e.g., Garleanu and Pedersen (2011), develop general asset pricing models by taking margins as exogenous. The endogenous determination of the margin in this model might inform the choice of the exogenous margins when applying these theories.

To establish the comparative statics for the type of belief disagreements, it is necessary first to define the different types. The following definition introduces a notion of skewness of optimism.

Definition 3 (Upside Skew of Optimism). The optimism of distribution $\tilde{F}_{1}$ is more skewed to the upside than $F_{1}$ if:

(a) The distributions yield the same valuation of the asset, that is, $E\left[s ; \tilde{F}_{1}\right]=E\left[s ; F_{1}\right]$.

(b) The hazard rates of $\tilde{F}_{1}$ and $F_{1}$ satisfy the (weak) single crossing condition:

$$
\left\{\begin{array}{l}
\frac{\tilde{f}_{1}(s)}{1-\tilde{F}_{1}(s)} \geq \frac{f_{1}(s)}{1-F_{1}(s)} \text { if } s<s^{R}, \\
\frac{\tilde{f}_{1}(s)}{1-\tilde{F}_{1}(s)} \leq \frac{f_{1}(s)}{1-F_{1}(s)} \text { if } s>s^{R},
\end{array} \text { for some } s^{R} \in S .\right.
$$

The optimism of distribution $\tilde{F}_{1}$ is strictly more skewed to the upside than $F_{1}$ if the inequalities in (b) are strict.

To interpret this definition, note that the distributions $\tilde{F}_{1}$ and $F_{1}$ cannot be compared according to the hazard rate order of assumption (A2). In addition, these distributions lead to the same valuation of the asset, that is, they have the same "level" of optimism. Note also that $\tilde{F}_{1}$ has a lower hazard rate than $F_{1}$ over the region $\left(s^{R}, s^{\max }\right)$. Thus, conditional on upside states, $s \geq s^{R}$, $\tilde{F}_{1}$ is weakly more optimistic than $F_{1}$ in the sense of assumption (A2). In contrast, $F_{1}$ has a lower 



Figure 2: The top two panels display the hazard rates for traders' priors in the two cases analyzed in Example 1. The bottom panel plots the corresponding equilibria.

hazard rate than $\tilde{F}_{1}$ over the downside region $\left(s^{\min }, s^{R}\right)$, and thus its optimism is concentrated more on this region. Hence, the optimism of $\tilde{F}_{1}$ is skewed to the upside.

The probability distributions, $F_{1, D}$ and $F_{1, U}$, for the two cases of Example 1 satisfy condition (18). In particular, the optimism of $F_{1, U}$ is weakly more skewed to the upside, as illustrated in Figure 2. The same figure also plots the equilibrium for the two cases and illustrates that the equilibrium price and loan riskiness corresponding to $F_{1, U}$ are higher. The next result shows that this observation is generally true.

Theorem 4 (Type of Disagreements). Consider the equilibrium characterized in Theorem 3. If optimism becomes more skewed (resp. strictly more skewed) to the upside, i.e., if $F_{1}$ is changed to $\tilde{F}_{1}$ that satisfies condition (18) while still satisfying assumption (A2), then: the asset price $p$ and the loan riskiness $\bar{s}^{*}$ weakly (resp. strictly) increase, and the margin $m$ weakly (resp. strictly) decreases.

This result formalizes the sense in which optimism is asymmetrically disciplined. I provide a sketch proof which is completed in Appendix A.3. Eq. (12) shows that the optimistic belief affects the optimality curve, $p^{\text {opt }}(\bar{s})$, through optimists' conditional valuation, $E_{1}[s \mid s \geq \bar{s}]$. The analysis in the appendix shows that $\tilde{E}_{1}[s \mid s \geq \bar{s}] \geq E_{1}[s \mid s \geq \bar{s}]$ for each $\bar{s} \in\left(s^{\min }, s^{\max }\right)$. That is, an increase in the upside skewness of optimism increases the conditional valuation for each $\bar{s}$, even though it does not increase the unconditional valuation. It follows that the optimality 

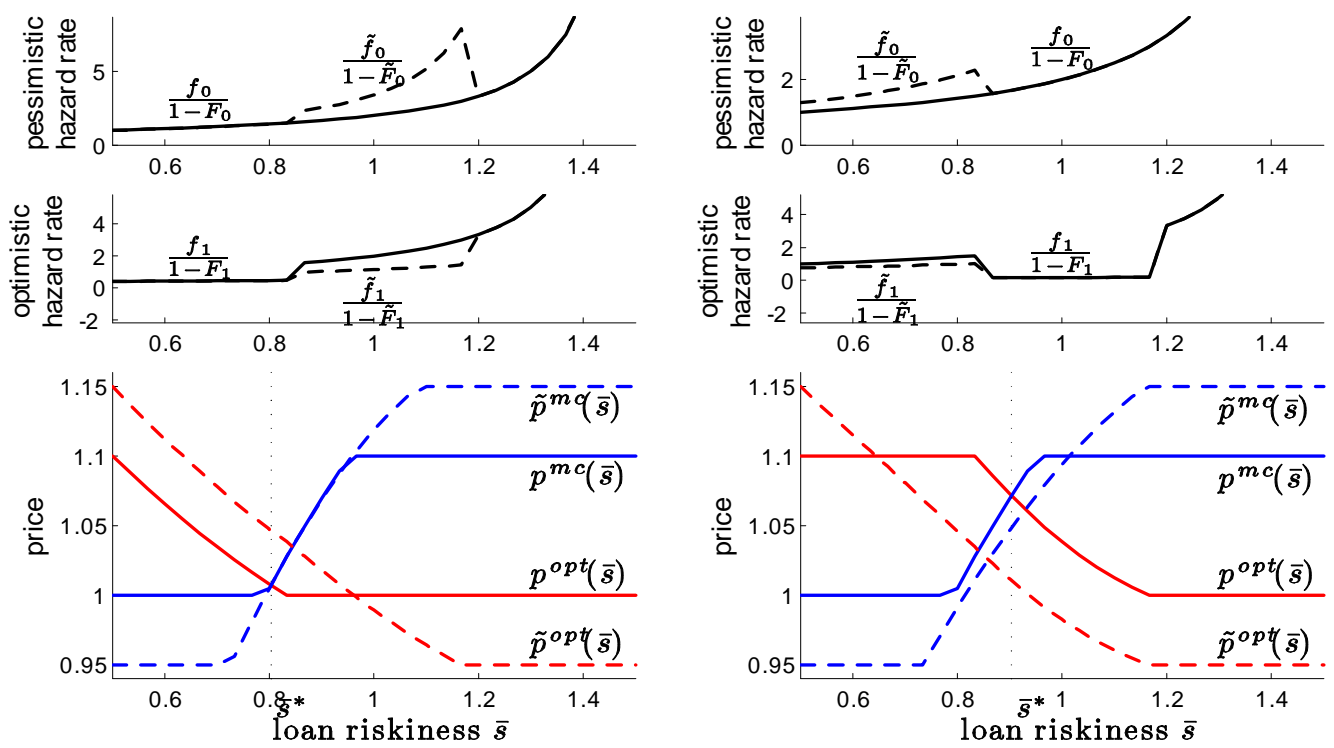

Figure 3: The left panel plots the equilibrium for the first case of Example 2, the increase in belief disagreements concerns states above $\bar{s}^{*}$ and it increases the asset price. The left panel plots the equilibrium for the second case of Example 2, the increase in belief disagreements concerns states below $\bar{s}^{*}$ and it decreases the asset price.

curve shifts up pointwise. Since the market clearing curve, $p^{m c}(\cdot)$, is unchanged [cf. Eq. (16)], the comparative statics in the theorem statement follow. Intuitively, as optimism becomes more concentrated on upside states, optimists' borrowing constraints become looser and their demand for the asset becomes greater. This leads to a low margin and a high equilibrium price.

Theorem 4 shows that the type of the belief disagreements has an unambiguous effect on margins and asset prices. A natural question is whether the level of belief disagreements has similar robust predictions. The answer is no, as illustrated by the following example.

Example 2 (Ambiguous Effects of an Increase in Disagreements). Consider the following two cases, each of which features an increase in the level of belief disagreements.

Case (i). Consider the first case of Example 1 with downside optimism. Suppose the beliefs are changed to $\tilde{F}_{0}$ and $\tilde{F}_{1}$ with density functions given by:

$$
\tilde{f}_{0}=\left\{\begin{array}{cl}
1 & \text { if } s \in \mathcal{S}_{B} \\
1+0.45 & \text { if } s \in \mathcal{S}_{N} \\
1-0.45 & \text { if } s \in \mathcal{S}_{G}
\end{array}, \quad \tilde{f}_{1}=\left\{\begin{array}{cl}
0.4 & \text { if } s \in \mathcal{S}_{B} \\
1.3-0.45 & \text { if } s \in \mathcal{S}_{N} \\
1.3+0.45 & \text { if } s \in \mathcal{S}_{G}
\end{array}\right.\right.
$$

That is, pessimists' probability for the normal event increases and their probability for the good event decreases, while the opposite happens to optimists' prior. As the right panel of Figure 3 shows, in this case, the increase in belief disagreements leads to an increase in the asset price.

Case (ii). Next consider the second case of Example 1 with upside optimism. Suppose the 
beliefs are changed to $\tilde{F}_{0}$ and $\tilde{F}_{1}$ with density functions given by:

$$
\tilde{f}_{0}=\left\{\begin{array}{cl}
1+2 \eta_{0} & \text { if } s \in \mathcal{S}_{B} \\
1-\eta_{0} & \text { if } s \in \mathcal{S}_{N} \\
1-\eta_{0} & \text { if } s \in \mathcal{S}_{G}
\end{array}, \quad \tilde{f}_{1}=\left\{\begin{array}{cl}
1\left(1-2 \eta_{1}\right) & \text { if } s \in \mathcal{S}_{B} \\
0.1\left(1+\eta_{1}\right) & \text { if } s \in \mathcal{S}_{N} \\
1.9\left(1+\eta_{1}\right) & \text { if } s \in \mathcal{S}_{G}
\end{array},\right.\right.
$$

for some parameters $\eta_{0}$ and $\eta_{1}$. That is, pessimists' probability for the bad event increases (and their relative probability for good and the normal event remains constant), while optimists' probability for the bad event decreases. In addition, choose parameters $\eta_{0}$ and $\eta_{1}$ such that both pessimists' and optimists' valuation of the asset is the same as in the first case of this example. As Figure 3 shows, in this case, the increase in belief disagreements decreases the asset price.

Miller (1977) had argued that, when short selling is constrained, an increase in belief disagreements tends to increase the overvaluation of the asset (relative to the average valuation in the population) because the asset is held by the most optimistic traders. Example 2 illustrates that, in this model, the increase in belief disagreements [in the sense of assumption (A2)] has no robust predictions for the asset price. This is because both optimists' and pessimists' beliefs affect the price. While an increase in borrowers' optimism tends to increase the price, a decrease in lenders' pessimism tends to decrease it by tightening the borrowing constraints. This observation illustrates that the Miller mechanism might not apply in markets in which optimists finance their asset purchases by borrowing from less optimistic traders.

I next derive a qualified version of the Miller mechanism for these markets. In particular, the next result shows that an increase in belief disagreements has robust predictions if the type of the additional increase is taken into account. In the first case of Example 2, the increase in belief disagreements is concentrated on states below the default threshold $\bar{s}^{*}$, which leads to a decrease in the asset price. In the second case of the example, the increase in belief disagreements is concentrated on states above the default threshold $\bar{s}^{*}$, which leads to an increase in the asset price increases (see also Figure 3). The next result establishes that these properties are general. Taken together, the results in this section suggest that what investors disagree about matters for asset prices, to a greater extent than the level of their disagreement.

Theorem 5 (Level of Disagreements). Consider the equilibrium characterized in Section 3. Consider an increase in belief disagreements, that is, suppose $F_{1}, F_{0}$ are changed to $\tilde{F}_{1}, \tilde{F}_{0}$ that satisfy:

$$
\frac{\tilde{f}_{1}(s)}{1-\tilde{F}_{1}(s)} \leq \frac{f_{1}(s)}{1-F_{1}(s)} \text { and } \frac{\tilde{f}_{0}(s)}{1-\tilde{F}_{0}(s)} \geq \frac{f_{0}(s)}{1-F_{0}(s)} \text { for } s \in S .
$$

(i) Suppose the increase in belief disagreements is concentrated on states above $\bar{s}^{*}$ in the sense that the hazard rate inequalities (19) are satisfied with equality for $s \in\left(s^{\mathrm{min}}, \bar{s}^{*}\right)$. Then, the asset price $p$ and the loan riskiness $\bar{s}^{*}$ weakly increase, and the margin $m$ weakly decreases.

(ii) Suppose the increase in belief disagreements is concentrated on states below $\bar{s}^{*}$ in the sense that the hazard rates inequalities (19) are satisfied with equality for $s \in\left(\bar{s}^{*}, s^{\max }\right)$. Then, the asset 
price $p$ weakly decreases and the margin $m$ weakly increases.

\section{$5 \quad$ Equilibrium with Short Selling}

The results so far share with Miller (1977) the assumption that short selling is not allowed, which restricts their applicability. This section shows that the main result, asymmetric disciplining, is in fact robust to allowing for short selling. I model short selling with the simple short contracts introduced in Section 3. I characterize the equilibrium with short contracts and establish a version of the asymmetric disciplining result for this setting.

It is useful to start by reviewing the salient features of a typical short sale transaction in financial markets. In a typical short sale, a trader borrows the asset from a lender who owns the asset. The borrower raises $p$ dollars from the short sale, which she leaves as cash-collateral with the lender. However, the lender requires additional protection, which induces the borrower to place an additional $m^{S} p$ dollars as cash-collateral. The short margin, $m^{S}$, is the analogue of the loan margin in the baseline setting since the borrower needs $m^{S} p$ dollars to short sell the asset. Next period, the lender rebates the borrower's cash collateral at a rebate rate, rebate, which is lower than the benchmark interest rate, $r=011$ The difference, $-r^{\text {rebate }}$, which is the analogue of the interest rate spreads in cash-loans, captures the fee received by the lender. In practice, the fee emerges from a variety of factors. Among other things, it compensates the lender for expected losses from a potential default on the short contract. The model below focuses purely on this component of the fee, abstracting away from other factors. The lender's total fee from the asset-loan can be written as:

$$
f=-r^{\text {rebate }}\left(1+m^{S}\right) p .
$$

Given the rebate rate and the total fee it implies, the net cash-collateral backing the borrower's promise to return the asset can be written as:

$$
\left(1+m^{S}\right) p\left(1+r^{\text {rebate }}\right)=\left(1+m^{S}\right) p-f
$$

The above short sale transaction can be captured in this model by the simple short contracts introduced in Section $2^{12}$

$$
\mathcal{B}^{S} \equiv\left\{\left([\varphi(s) \equiv s]_{s \in S}, 0, \gamma\right) \mid \gamma \in \mathbb{R}_{+}\right\}
$$

In particular, a simple short contract promises a replica of the asset collateralized by $\gamma$ units of

\footnotetext{
${ }^{11}$ In this model, the rebate rate is always negative in view of the normalization, $r=0$. In practice, the rebate rate is typically positive but lower than the benchmark interest rate. The assets with low or negative rebate rates, equivalently high lending fees, are said to be "special." See D'Avolio (2002) or Lamont (2004) for excellent descriptions of the short market.

${ }^{12}$ Recall that a simple short contract is generally denoted by $\beta=\left([\varphi(s)=\varphi s]_{s \in S}, \psi^{a}, \psi^{c}\right)$ for some $\varphi \in \mathbb{R}_{+}$. The set, $\mathcal{B}^{S}$, features two restrictions which are without loss of generality. First, I assume $\psi^{a}=0$ because the transfer implied by the contract, $\left(\varphi s, \psi^{a}, \psi^{c}\right)$, can be equivalently captured by the contract, $\left(\max \left(\varphi-\psi^{a}\right) s, 0, \psi^{c}\right)$. Second, I normalize the contracts by taking the number of assets short sold to be 1 .
} 
cash. To map this short contract to a short sale, let $m^{S}$ and $f$ be given as the solutions to the following equations:

$$
\gamma=\left(1+m^{S}\right) p-f \text { and } p-q(\gamma)=f .
$$

In particular, the cash-collateral of the short contract corresponds to the net cash-collateral, 20), posted in the short sale transaction. The difference between the price of the asset and the price of its replica (i.e., the short contract) corresponds to the total fee received by the lender. Given $m^{S}$ and $f$, the rebate rate, $r^{\text {rebate }}$, is also uniquely determined. Consequently, both the short margin and the total lending fee, as well as the rebate rate, will be endogenously determined in general equilibrium.

I next characterize the equilibrium corresponding to the set $\mathcal{B}^{S}$. In particular, to isolate the effects of short selling, I rule out the simple debt contracts analyzed in the baseline setting (see appendix A.5 for a generalization of the results in this section to a setting with both simple debt and short contracts). The analysis of equilibrium follows closely Section 3 . In this case, the equivalent principal-agent equilibrium is one in which pessimists (who are the borrowers with asset-loans) choose the short contract subject to optimists' participation constraint. Since debt contracts are not available, optimists are indifferent between making an unleveraged investment in the asset and buying the short contracts sold by pessimists.

The key observation is that pessimists default on the short contract when the future asset price, $s$, exceeds the value of the cash-collateral, $\gamma$. Thus, the actual payoff of the short contract is given by $\min (\gamma, s)$, and the price of the short contract that makes optimists indifferent can be written as:

$$
q(\gamma)=\frac{1}{\frac{E_{1}[s]}{p}} E_{1}[\min (\gamma, s)] .
$$

Note that the price is increasing in the cash-collateral. Equivalently, keeping $p$ fixed, the lending fee in 22 is decreasing in $\gamma$. When the cash-collateral is high, the short contract defaults less often and the lending fee, which compensates the lender for losses from default, is lower ${ }^{13}$

Next suppose pessimists use all of their cash holdings as collateral in short contracts. Pessimists choose the cash-collateral, $\gamma$, and the quantity, $x_{0}$, of its short contracts to solve:

$$
\begin{aligned}
\max _{\left(x_{0}, \gamma\right) \in \mathbb{R}_{+}^{2}} & x_{0} \gamma-x_{0} E_{0}[\min (\gamma, s)], \\
\text { s.t. } & x_{0} \gamma=n_{0}+x_{0} \frac{1}{\frac{E_{1}[s]}{p}} E_{1}[\min (\gamma, s)] .
\end{aligned}
$$

The first line is pessimists' expected return which consists of their return from cash holdings net of their expected payments on short contracts. The second line is pessimists' budget constraint which incorporates optimists' participation constraint. The budget constraint illustrates that

\footnotetext{
${ }^{13}$ For example, the cash-collateral $\gamma=s^{\max }$ leads to $q(\gamma)=p$ and a lending fee $f=0$, because this short contract is completely safe. From Eq. 22 , the margin on this contract is solved from $m^{s h} p=s^{\max }-p$. Hence, this contract corresponds to the safe short contract analyzed in Gromb and Vayanos (2002). The difference in this paper is that riskier short contracts (with lower short margins) are also available for trade.
} 
choosing lower cash-collateral enables pessimists to sell a greater number of short contracts. On the other hand, recall that higher cash-collateral leads to a lower lending fee. Thus, pessimists face a trade-off between a greater short position and a lower lending fee.

The next result, which is the main result of this section, characterizes pessimists' optimal short contract. The result requires the following analogues of assumptions (A1) and (A2):

Assumption $\left(\mathbf{A 1}^{S}\right) \cdot n_{1}>E_{0}[s]\left(1+\frac{n_{0}}{s^{\max }-E_{0}[s]}\right)$.

Assumption $\left(\mathbf{A 2}^{S}\right)$. The probability distributions $F_{1}$ and $F_{0}$ satisfy ${ }^{14}$

$$
\frac{f_{1}(s)}{\int_{s^{\min } \tilde{s} d F_{1}}^{s}}>\frac{f_{0}(s)}{\int_{s^{\min }}^{s} \tilde{s} d F_{0}} \text { for each } s \in\left(s^{\min }, s^{\max }\right)
$$

Theorem 6 (Asymmetric Disciplining of Pessimism). Suppose the contract space is restricted to simple short contracts, $\mathcal{B}^{T}=\mathcal{B}^{S}$, and assumptions $\left(A 1^{S}\right)$ and $\left(A 2^{S}\right)$ hold. Fix asset price $p \in\left(E_{0}[s], E_{1}[s]\right)$, and consider pessimists' problem (8). The cash-collateral, $\gamma$, of the optimal short contract is the unique solution to the following equation over the range $\left(s^{\min }, s^{\max }\right)$ :

$$
p=p^{o p t, S}(\gamma) \equiv \frac{E_{1}[s]}{F_{0}(\gamma) \frac{\int_{s \min }^{\gamma} s d F_{1}}{\int_{s \min }^{\gamma} s d F_{0}}+1-F_{1}(\gamma)} .
$$

The pricing formula (25) shows that pessimism is asymmetrically disciplined in equilibrium. In particular, the pessimistic belief enters the relation only through its effect on the following expression (which increases the price): $\int_{s^{\min }}^{\gamma} \frac{d F_{0}}{F_{0}(\gamma)}$. This expression shows that pessimism about the relative likelihood of downside states, $s \leq \gamma$, decreases the asset price. More importantly, it also shows that any other type of pessimism does not decrease the asset price. For example, pessimism about the relative likelihood of upside states, $s \geq \gamma$, does not affect the price. Similarly, pessimism about the probability of the event $\{s \geq \gamma\}$ (while keeping the relative likelihood of states $s \leq \gamma$ fixed) does not decrease the price. Figure 4 illustrates this result by plotting the optimality curve, $p^{\text {opt,S}}(\gamma)$, for two examples that differ only in the type of pessimism. It shows that the asset price is greater when the pessimism on the upside than when it is on the downside.

The intuition for this result closely parallels the intuition for the asymmetric disciplining of optimism (cf. Section 3.3). First consider why $p^{o p t, S}(\gamma)$ is decreasing, that is, why pessimists choose lower cash-collateral when the price is higher. Given a higher $p$, pessimists have a greater incentive to bet. Consequently, they choose lower $\gamma$ because this enables them to increase their short position [cf. problem (24)]. Next, to see the intuition for asymmetric disciplining, consider the cash-collateral, $\gamma=1.1$, for the two examples plotted in Figure 4 . Consider how much the price should increase (from the pessimistic valuation, $E_{0}[s]$ ) for pessimists to short sell with $\gamma$. The answer depends on the type of pessimism. When the pessimism is on the upside (for states $s \geq \gamma)$, then optimists charge a positive short fee because they think the short contract is likely to

\footnotetext{
${ }^{14}$ Assumptions (A2) and $\left(\mathrm{A} 2^{S}\right)$ do not imply each other. However, Assumption (A2 ${ }^{S}$ ) [as well as assumption (A2)] is implied by the monotone likelihood ratio property (cf. Section 3.1).
} 



Figure 4: The top two panels display the pessimistic and the optimistic pdf's for two examples. The examples differ only in the type of pessimism: The bold lines correspond to pessimism on the downside while the dashed lines correspond to pessimism on the upside. The bottom panel plots the corresponding equilibria (the intersection of the optimality and market clearing curves).

default. Moreover, this short fee appears too high to pessimists because they think the contract is unlikely to default. Consequently, the price should increase considerably to entice pessimists to short sell with $\gamma$. In contrast, when the pessimism is concentrated on the downside, optimists and pessimists disagree less about the short fee corresponding to cash-collateral $\gamma$. Consequently, the price does not need to increase as much to entice pessimists to short sell with $\gamma$. Put differently, in this case pessimists face looser constraints for short selling, which leads to a lower price.

The equilibrium price is characterized by asset market clearing. Optimists' endowment is spent to purchase the asset and the short contracts sold by pessimists. Thus, asset market clearing implies: $n_{1}=p+x_{0} q(\gamma)$. Using Eqs. (22), (23), (24), this condition can be written as:

$$
\frac{n_{1}}{p}=1+\frac{n_{0}}{p} \frac{1}{m^{S}} \frac{E_{1}[\min (\gamma, s)]}{E_{1}[s]} .
$$

The left hand side of this expression corresponds to the demand for the asset. The right hand side corresponds to the effective supply, which is greater than the physical supply (one unit) in view of short selling. Note that a smaller short margin, $m^{S}$, enables pessimists to short sell a greater number of assets ${ }^{15}$ After substituting for $m^{S}$ in terms of $p$ and $\gamma$, Eq. (26) implicitly defines a market clearing relation between the price and the cash collateral, $p^{m c, S}(\gamma)$. Moreover, Appendix A.4 shows that $p^{m c, S}(\gamma)$ is increasing in $\gamma$. Intuitively, a smaller cash-collateral leads to a smaller

\footnotetext{
${ }^{15}$ The last term in Eq. [26), $\frac{E_{1}[\min (\gamma, s)]}{E_{1}[s]}$, is a normalizing factor which emerges from the fact that the short contract is only an imperfect replica of the asset.
} 
short margin, which in turn leads to a greater short position and a lower market clearing price. The equilibrium price and cash-collateral pair, $(p, \gamma)$, is determined as the unique intersection of the optimality curve, $p^{o p t, S}(\gamma)$ and the market clearing curve, $p^{m c, S}(\gamma)$ (see Figure 4).

Note that the asymmetric disciplining of pessimism identified in this section is complementary to the asymmetric disciplining of optimism. When belief disagreements are on the upside, optimists are less constrained while pessimists are more constrained. In equilibrium, this leads to lower loan margins and higher short margins, and an asset price closer to the optimistic valuation. In contrast, when belief disagreements are on the downside, loan margins are higher, short margins are lower, and the price is closer to the pessimistic valuation. These results establish that the main asymmetric disciplining result is robust to allowing for short selling.

\section{Equilibrium with Richer Contracts}

While simple debt and short contracts are common in financial markets, rapid financial innovation in recent years has made available richer contracts such as options, futures, and more complex derivatives. A natural question is how the availability of these contracts affect optimists' or pessimists' borrowing constraints. Conversely, one might also wonder whether the borrowing constraints among traders with disagreements might be a driving force behind some of the recent innovations. To address these questions, this section considers the equilibrium with the unrestricted contract space. The analysis is simplified by the fact that, when the contract space is sufficiently rich, every Arrow-Debreu equilibrium corresponds to a general equilibrium. Characterizing the unique Arrow-Debreu equilibrium reveals two main results. First, a version of the asymmetric disciplining result also holds for this setting. Second, relatively complex contracts that resemble some of the recent innovations endogenously emerge in equilibrium. The analysis also shows that richer contracts on balance have an ambiguous effect on the asset price.

I first define the Arrow-Debreu (AD) equilibrium for this economy. Suppose for each state $s$ there is an AD security that pays 1 dollar is the state $s$ realized and nothing otherwise. I model the price of $\mathrm{AD}$ securities with a (Lebesgue) measurable and bounded function $q^{A D}: S \rightarrow \mathbb{R}_{+}$. Traders choose measurable functions $z_{i}: S \rightarrow \mathbb{R}_{+}$, which correspond to their positions in these securities. As before, traders also choose their asset and cash holdings, $a_{i}$ and $c_{i}$. Thus, their problem is:

$$
\begin{aligned}
\max _{\left(a_{i}, c_{i},\right) \in \mathbb{R}_{+}^{2}, z_{i}: S \rightarrow \mathbb{R}} & \int_{S}\left(a_{i} s+c_{i}+z_{i}(s)\right) d F_{i}, \\
\text { s.t. } \quad & a_{i} p+c_{i}+\int_{S} z_{i}(s) q^{A D}(s) d s \leq n_{i}, \\
& a_{i} s+c_{i}+z_{i}(s) \geq 0 \text { almost everywhere (a.e.) on } S .
\end{aligned}
$$


Definition 4. An Arrow-Debreu equilibrium is a collection of prices $\left(p \in \mathbb{R}_{+}, q^{A D}: S \rightarrow \mathbb{R}_{+}\right)$ and portfolios $\left(a_{i}^{*}, c_{i}^{*}, z_{i}^{*}(\cdot)\right)_{i \in\{0,1\}}$ such that the portfolios solve problem (27) for each $i \in\{0,1\}$, the asset market clears, $\sum_{i \in\{0,1\}} a_{i}^{*}=1$, and debt markets clear, $\sum_{i \in\{0,1\}} z_{i}^{*}(s)=0$ a.e. on $S$.

The key feature of the AD equilibrium is that all promises are enforced at the balance sheet level (via an unspecified mechanism, e.g., the threat of punishment) subject to a nonnegative consumption constraint for the borrower. In contrast, promises in the general equilibrium are enforced by the collateral explicitly specified in each borrowing contract. Hence, loosely speaking, the general equilibrium features tighter constraints on borrowing. Nonetheless, the next result shows that, when the contract space is sufficiently rich, every AD equilibrium for this economy corresponds to a general equilibrium.

Theorem 7 (Equivalence of the AD Equilibrium to a General Equilibrium). Suppose there exists an $A D$ equilibrium, $\left[p^{*}, q^{A D}(\cdot),\left(a_{i}^{*}, c_{i}^{*}, z_{i}^{*}\right)\right]$, and the contract space $\mathcal{B}^{T}$ is either unrestricted, $\mathcal{B}^{T}=\mathcal{B}$, or it contains the following two contracts, $\left\{\left(\boldsymbol{\varphi}_{i}, \alpha_{i}, \gamma_{i}\right)\right\}_{i \in\{0,1\}}$, where:

$$
\boldsymbol{\varphi}_{i}(s) \equiv \max \left(0,-z_{i}^{*}(s)\right) \text { for each } s \in S, \quad \alpha_{i} \equiv a_{i}^{*} \text { and } \gamma_{i} \equiv c_{i}^{*}
$$

Then, there exists a general equilibrium, $\left[(\hat{p},[\hat{q}(\cdot)]),\left(\hat{a}_{i}, \hat{c}_{i}, \hat{\mu}_{i}^{+}, \hat{\mu}_{i}^{-}\right)\right]$, that is equivalent to the $A D$ equilibrium. In particular, prices are given by:

$$
\hat{p}=p^{*} \text { and } \hat{q}((\varphi, \alpha, \gamma))=\int_{S} \min (\alpha s+\gamma, \varphi(s)) q^{A D}(s) d s
$$

and portfolios are given by: $\left(\hat{a}_{i}, \hat{c}_{i}\right)=\left(a_{i}^{*}, c_{i}^{*}\right)$ and each $\hat{\mu}_{i}^{-}$is a Dirac measure that puts weight only at contract $\left(\varphi_{i}, \alpha_{i}, \gamma_{i}\right)$ (and $\hat{\mu}_{1}^{+}=\hat{\mu}_{0}^{-}$and $\left.\hat{\mu}_{0}^{+}=\hat{\mu}_{1}^{-}\right)$.

The constructed general equilibrium is equivalent to the $\mathrm{AD}$ equilibrium in the sense that the promises are equivalent [cf. Eq. (28)] and debt contracts are priced according to their fundamental values in the $\mathrm{AD}$ equilibrium [cf. Eq. 229]. Intuitively, the general equilibrium features a more constrained choice set for traders than the AD equilibrium. Thus, a portfolio that is optimal in the latter equilibrium is also optimal in the former equilibrium (with corresponding prices) as long as it is feasible. This feasibility is in turn ensured when the contract space is sufficiently rich.

I next characterize the AD equilibrium. Given the availability of the asset and cash, in addition to the $\mathrm{AD}$ securities, there are two degrees of freedom in traders' portfolios, $\left(a_{i}, c_{i}, z_{i}(\cdot)\right)$. Thus, without loss of generality, suppose all units of the asset are held by optimists, i.e., $a_{0}=0$, and all units of cash are held by pessimists, i.e., $c_{1}=0$. This convention ensures continuity with the earlier sections in which optimists invest in the asset and pessimists invest in cash. Under this convention, optimists finance their asset purchases in part by selling borrowing contracts to pessimists, and pessimists finance their cash purchases in part by selling borrowing contracts to optimists. In addition, suppose the following analogues of assumptions (A1) and (A2) hold:

Assumption $\left(\mathbf{A 1}^{R}\right) \cdot n_{1}+n_{0}>E_{1}[s]$. 
Assumption (MLRP). The probability distributions $F_{1}$ and $F_{0}$ satisfy the monotone likelihood ratio property: that is, $\frac{f_{1}(s)}{f_{0}(s)}$ is strictly increasing over $S$.

The first assumption ensures that traders' endowments are sufficiently large to purchase the entire asset supply. The second assumption, which is stronger than both (A2) and (A2 ${ }^{S}$ ), ensures that the $\mathrm{AD}$ equilibrium takes a simple form. In particular, traders' $\mathrm{AD}$ security positions satisfy:

$$
z_{1}(s) \equiv\left\{\begin{array}{cl}
-a_{1} s & \text { a.e. on }\{s \in S \mid s<\bar{s}\} \\
c_{0} & \text { a.e. on }\{s \in S \mid s>\bar{s}\}
\end{array} \text { and } z_{0}(s)=\left\{\begin{array}{cc}
a_{1} s & \text { a.e. on }\{s \in S \mid s<\bar{s}\} \\
-c_{0} & \text { a.e. on }\{s \in S \mid s>\bar{s}\}
\end{array}\right. \text {. }\right.
$$

for some $\bar{s} \in\left(s^{\min }, s^{\max }\right)$. That is, optimists sell all of the asset payoff in states $s<\bar{s}$ and keep it in states $s>\bar{s}$. They also buy all of the cash payoff in states $s>\bar{s}$ via the AD securities sold by pessimists. Consequently, optimists receive all of the endowment in the economy in states $s>\bar{s}$, and they receive nothing in states $s<\bar{s}$. Symmetrically, pessimists receive all of the economy's endowment in states $s<\bar{s}$. Intuitively, traders of each type concentrate their payoffs in the states in which they find the most likely.

In addition, $\mathrm{AD}$ security prices also take a simple form. To see this, let $R_{i} \geq 1$ denote the Lagrange multiplier on the budget constraint of problem (27), which captures type $i$ traders' gross return on net worth. The analysis in the appendix shows that $q^{A D}(s)=\max \left(\frac{f_{0}(s)}{R_{0}}, \frac{f_{1}(s)}{R_{1}}\right)$ a.e. on $S$, which in turn implies:

$$
q^{A D}(s)=\left\{\begin{array}{ll|l}
\frac{f_{0}(s)}{R_{0}}, & \text { a.e. on }\{s \in S & s<\bar{s}\} \\
\frac{f_{1}(s)}{R_{1}}, & \text { a.e. on }\{s \in S & s<\bar{s}\}
\end{array}, \text { where } \frac{f_{0}(\bar{s})}{f_{1}(\bar{s})}=\frac{R_{0}}{R_{1}} .\right.
$$

Intuitively, traders of each type price the $\mathrm{AD}$ securities in the region in which they receive a positive payoff. Furthermore, to rule out arbitrage, the price of the asset and cash satisfy:

$$
\begin{aligned}
p & =\frac{1}{R_{0}} \int_{s^{\min }}^{\bar{s}} s f_{0}(s) d s+\frac{1}{R_{1}} \int_{\bar{s}}^{s^{\max }} s f_{1}(s) d s \\
\text { and } \quad 1 & =\frac{1}{R_{0}} F_{0}(\bar{s})+\frac{1}{R_{1}}\left(1-F_{1}(\bar{s})\right) .
\end{aligned}
$$

Finally, market clearing requires optimists' asset demand to be $a_{1}=1$ and pessimists' cash demand to be $c_{0}=n_{1}+n_{0}-p$. Moreover, since pessimists consume all of the economy's endowment in states $s<\bar{s}$, market clearing in $\mathrm{AD}$ securities requires:

$$
n_{0}=\frac{1}{R_{0}} \int_{s^{\min }}^{\bar{s}}\left(s+\left(n_{1}+n_{0}-p\right)\right) f_{0}(s) d s .
$$

The following result completes the characterization and establishes the uniqueness of equilibrium.

Theorem 8 (Arrow-Debreu Equilibrium). Suppose assumptions (A1 ${ }^{R}$ ) and (MLRP) hold. Then, there exists an $A D$ equilibrium, $\left[\left(p, q^{A D}\right),\left(a_{i}^{*}, c_{i}^{*}, z_{i}^{*}(\cdot)\right)\right]$ in which $a_{1}^{*}=1$ and $c_{0}^{*}=n_{1}+$ $n_{0}-p$ (and $a_{0}^{*}=c_{1}^{*}=0$ ). The AD security holdings and prices are respectively given by Eqs. (30) and (31) for some threshold state, $\bar{s}^{*} \in\left(s^{\min }, s^{\max }\right)$, and returns $R_{0}^{*}, R_{1}^{*}$. The asset price, $p$, 
along with $\left(\bar{s}^{*}, R_{0}^{*}, R_{1}^{*}\right)$ are characterized as the unique solution to the four equations (31) - (34). The equilibrium asset price satisfies $p \in\left(E_{0}[s], E_{1}[s]\right)$. The equilibrium is essentially unique in the sense that $p$ is uniquely determined; and the $A D$ security prices, $q^{A D}(s)$, and traders' consumption, $a_{i} s+c_{i}+z_{i}(s)$, are uniquely determined a.e. on $S$.

Theorem 8 illustrates the main results of this section. First, a version of the asymmetric disciplining result continues to apply with richer contracts. In particular, Eqs. (31) - (34) show that optimism about the relative likelihood of downside states, $s<\bar{s}$, has no effect on the asset price, $p$, because these states are effectively priced by pessimists. In contrast, the more detailed characterization in the appendix, Eqs. A.27 - A.28, show that optimism about the relative likelihood of upside states, $s>\bar{s}$, increases the price.

Second, Eq. (30) illustrates that relatively complex contracts that resemble some of the recent financial innovations in the US mortgage market endogenously emerge to facilitate betting. In particular, the asset is tranched so that optimists hold the payoff in states $s>\bar{s}$, while selling it to pessimists in states $s<\bar{s}$. The tranching of the asset resembles collateralized debt and mortgage obligations (CDOs and CMOs), which were introduced into the US mortgage market in the last three decades. The role of these derivatives, just like the role of tranching in the model, is to split the payoff from the underlying asset across heterogeneous traders. Similarly, Eq. (30) also illustrates that cash is also tranched so that pessimists hold the payoff in states $s<\bar{s}$, while optimists hold it in states $s>\bar{s}$. The tranching of cash resembles credit default swaps (CDSs) or synthetic CDOs which were introduced into the mortgage market in the run-up to the recent subprime crisis. These insurance arrangements, just like the tranching of the cash in the model, enable some traders (pessimists in the model) to receive payments in bad states while paying a premium in good states. Moreover, these contracts arguably played a role in facilitating speculation in the run-up to the recent crisis by enabling pessimists to take positions in line with their negative views on the housing market (cf. Lewis, 2010). Taken together, this analysis creates a presumption that belief disagreements (or a similar source of heterogeneity in valuations) could be a driving force behind some of the recent financial innovations in the US mortgage market (see Fostel and Geanakoplos, 2012, for a similar analysis).

A natural question is how the availability of richer contracts affects the asset price, $p$. Example 3 in Appendix A.6 shows that the answer is in general ambiguous. In particular, the AD equilibrium price might be higher or lower than the price in the baseline setting with only simple debt contracts. Intuitively, richer contracts create two forces that push in opposite directions. On the one hand, tranching of the asset enables traders to split the asset payoff more "efficiently," in the sense that traders receive all of the payoff in the states in which they find the most likely. This effect tends to increase the value of the asset (although it also creates other general equilibrium effects). On the other hand, tranching of cash similarly enables traders to split the payoff from cash more efficiently, which tends to increase the value of cash relative to the asset, and thus, to reduce the asset price. Consequently, the net effect on the asset price is ambiguous and depends (among other things) on traders' endowments, $n_{1}$ and $n_{0}$. 


\section{Conclusion}

This paper theoretically analyzed the effect of belief disagreements on asset prices and financial contracts. The central feature of the model is that traders borrow by selling collateralized contracts to lenders who do not share the same beliefs. In particular, the lenders do not value the collateral as much as the borrowers do, which represents an endogenous borrowing constraint. I have considered the effect of this constraint in a number of settings that differ in the types of collateralized contracts that are available for trade.

In the baseline setting, I have restricted attention to simple debt contracts, which are useful to analyze optimists' borrowing constraints. I have also considered an extension with simple short contracts, which facilitate the analysis of pessimists' borrowing constraints. These analyses have established that both optimism and pessimism are asymmetrically disciplined by the endogenous borrowing constraint. In particular, the tightness of the constraint depends on the nature of belief disagreements. When belief disagreements are on the upside, optimists are less constrained while pessimists are more constrained, which leads to relatively low loan margins, relatively high short margins, and an asset price closer to the optimistic valuation. When belief disagreements are on the downside, the situation is the opposite.

These results suggest that what investors disagree about matters for asset prices. A large and growing empirical literature, motivated by Miller's (1977) theoretical findings, has shown that accounting for the level of belief disagreements can considerably improve our understanding of asset prices (e.g., Chen, Hong and Stein, 2001, Yu, 2011, Hong and Sraer, 2012). My paper suggests that a fruitful future research direction could be to empirically investigate the effect of the type of belief disagreements. To give an example of this approach, consider the value effect in stock returns. Stocks with high ratios of book value to market value, value stocks, have historically delivered higher average returns (after controlling their beta with the market portfolio) relative to stocks with low book-to-market ratios, growth stocks. Various explanations have been offered for this phenomenon, which is one of the "puzzles" of the risk-based asset pricing literature. A prominent non-risk-based explanation is that growth stocks are associated with greater optimism because some investors extrapolate too much from the recent good performance of these stocks (cf. Lakonishok, Shleifer, and Vishny, 1994). My paper offers a related explanation under the plausible assumption that growth stocks are associated with more upside optimism relative to value stocks (because they are associated with more uncertainty about upside states). This difference in the type of optimism would increase the price of growth stocks, and reduce their expected returns, even if investors have the same level of optimism for both growth and value stocks. The key insight is that endogenous borrowing constraints are looser for optimists (and tighter for pessimists) when optimism is on the upside, which makes the marginal trader in growth stocks endogenously more optimistic. Consistent with this prediction, Yu (2011) shows that an increase in belief disagreements about growth stocks (measured by the dispersion of analysts' forecasts about long run earnings growth) is associated with a greater price increase compared to a similar increase in belief disagreements about value stocks. 
My paper also generates testable implications regarding financial innovation. An extension of the model with richer contracts has revealed that relatively complex contracts, which resemble some of the recent financial innovations (in particular, CDOs, CMOs, and CDSs), endogenously emerge in equilibrium. These results suggest that belief disagreements (or a similar source of heterogeneity in valuations) could provide one explanation for the rapid financial innovation in the US mortgage market in recent years.

For tractability, the analysis has been carried out with risk neutral traders and two belief types. The results are robust to allowing for moderate amounts of risk aversion. The main effect of risk aversion is to provide an additional disciplining mechanism: Optimists are more reluctant to make leveraged investments because this increases their portfolio risks. Consequently, they choose safer debt contracts with higher margins relative to the risk-neutral case.

The results are also robust to allowing for more than two belief types. However, the model with a greater number of types yields additional insights. In particular, in this case traders are endogenously divided into three groups such that: the most pessimistic group invests in cash, the second most pessimistic group lends to optimists, and the optimistic group borrows to make leveraged investments in the asset. Importantly, when there are more than two continuation states (and regularity conditions are satisfied), borrowers and lenders within the groups are assortatively matched to one another according to their optimism. More specifically, the borrowers that are relatively more optimistic borrow from the lenders that are relatively more optimistic. Moreover, the relatively more optimistic pairs use loans with lower margins. Put differently, this version of the model generates the interesting implication that debt contracts with identical collateral but heterogeneous margins are simultaneously traded in equilibrium. A model along these lines is analyzed by Fostel and Geanakoplos (2011).

The analysis in this paper has not allowed debt contracts to be used as collateral. This is without loss of generality for the economy with two belief types considered here, but not necessarily for economies with more belief types. For example, with three belief types, I conjecture that the equilibrium will feature a pyramiding arrangement (defined in Geanakoplos, 1997): The trader with intermediate beliefs will lend to optimists by buying their debt contracts, and it will borrow from extreme pessimists by using these debt contracts as collateral. This arrangement is interesting because it captures key features of housing-related credit markets. To give an example, senior tranches of subprime CDOs (collateralized debt obligations) are debt contracts backed by subprime mortgage backed securities, which are themselves debt contracts backed by subprime mortgages, which are themselves debt contracts backed by houses. Understanding the nature of pyramiding, and its impact on asset prices, is a fascinating topic which I leave for future work. 


\section{A Appendices: Omitted Proofs and Extensions}

\section{A.1 Principal-Agent Equilibrium with Simple Debt Contracts}

This appendix completes the characterization of the principal-agent equilibrium analyzed in Section 3.3. The following lemma establishes some properties which are useful for the characterization. The rest of the appendix proves Theorems 2 and 3 .

Lemma 1. Suppose optimists' and pessimists' beliefs satisfy assumption (A2).

(i) Optimists' perceived interest rate $1+r_{1}^{p e r}(\bar{s})=\frac{E_{1}[\min (s, \bar{s})]}{E_{0}[\min (s, \bar{s})]}$ [cf. Eq. (14)] is strictly increasing in $\bar{s}$ over the range $\left(s^{\min }, s^{\max }\right)$. In particular, $r_{1}^{\text {per }}(\bar{s})>0$ for each $\bar{s}>s^{\text {min }}$.

(ii) $p^{\text {opt }}(\bar{s})$ is continuously differentiable and strictly decreasing, i.e., $\frac{d p^{\text {opt }}(\bar{s})}{d \bar{s}}<0$ over the range $\left(s^{\min }, s^{\max }\right)$. Moreover, $p^{\text {opt }}\left(s^{\min }\right)=E_{1}[s]$ and $p^{\text {opt }}\left(s^{\max }\right)=E_{0}[s]$.

Proof of Lemma 1. Part (i). First note that $\frac{d E_{i}[\min (s, \bar{s})]}{d \bar{s}}=1-F_{i}(\bar{s})>0$. Using this expression, the derivative of $1+r_{1}^{p e r}(\bar{s})=\frac{E_{1}[\min (s, \bar{s})]}{E_{0}[\min (s, \bar{s})]}$ can be written as:

$$
\frac{d\left(1+r_{1}^{p e r}(\bar{s})\right)}{d \bar{s}}=\frac{\left(1-F_{1}(\bar{s})\right) E_{0}[\min (s, \bar{s})]-E_{1}[\min (s, \bar{s})]\left(1-F_{0}(\bar{s})\right)}{\left(E_{0}[\min (s, \bar{s})]\right)^{2}} .
$$

Thus, to prove that $1+r_{1}^{\text {per }}(\bar{s})$ is increasing, it suffices to show that $\frac{E_{1}[\min (s, \bar{s})]}{E_{0}[\min (s, \bar{s})]}<\frac{1-F_{1}(\bar{s})}{1-F_{0}(\bar{s})}$ for each $\bar{s} \in\left(s^{\min }, s^{\max }\right)$. To prove this, note that for each $\bar{s} \in\left(s^{\min }, s^{\max }\right)$,

$$
\begin{aligned}
\frac{E_{1}[\min (s, \bar{s})]}{E_{0}[\min (s, \bar{s})]} & =\frac{\int_{s^{\min }}^{\bar{s}} s d F_{1}+\bar{s}\left(1-F_{1}(\bar{s})\right)}{\int_{s^{\min }}^{\bar{s}} s d F_{0}+\bar{s}\left(1-F_{0}(\bar{s})\right)} \\
& <\frac{\int_{s^{\min }}^{\bar{s}} s \frac{1-F_{1}(s)}{1-F_{0}(s)} d F_{0}+\bar{s}\left(1-F_{1}(\bar{s})\right)}{\int_{s^{\min }}^{\bar{s}} s d F_{0}+\bar{s}\left(1-F_{0}(\bar{s})\right)} \\
& <\frac{\int_{s^{\min }}^{\bar{s}} s d F_{0} \frac{1-F_{1}(\bar{s})}{1-F_{0}(\bar{s})}+\bar{s}\left(1-F_{1}(\bar{s})\right)}{\int_{s^{\min }}^{\bar{s}} s d F_{0}+\bar{s}\left(1-F_{0}(\bar{s})\right)}=\frac{1-F_{1}(\bar{s})}{1-F_{0}(\bar{s})},
\end{aligned}
$$

where the first inequality uses the hazard rate inequality $(9)$ and the second inequality uses the fact that $\frac{1-F_{1}(s)}{1-F_{0}(s)}$ is strictly increasing.

Part (ii). Using Eq. (11), note that

$$
\begin{aligned}
\frac{d p^{o p t}(\bar{s})}{d \bar{s}} & \equiv \bar{s} f_{0}(\bar{s})+\left(-f_{0}(\bar{s})+f_{1}(\bar{s}) \frac{1-F_{0}(\bar{s})}{1-F_{1}(\bar{s})}\right)\left(\int_{\bar{s}}^{s^{\max }} s \frac{d F_{1}}{1-F_{1}(\bar{s})}\right)-\frac{1-F_{0}(\bar{s})}{1-F_{1}(\bar{s})} \bar{s} f_{1}(\bar{s}) \\
& =-\left(1-F_{0}(\bar{s})\right)\left(\frac{f_{0}(\bar{s})}{1-F_{0}(\bar{s})}-\frac{f_{1}(\bar{s})}{1-F_{1}(\bar{s})}\right)\left(E_{1}[s \mid s \geq \bar{s}]-\bar{s}\right) .
\end{aligned}
$$

Here, the first line applies the chain rule while the second line substitutes $E_{1}[s \mid s \geq \bar{s}]$ and rearranges terms. The term, $\left(\frac{f_{0}(\bar{s})}{1-F_{0}(\bar{s})}-\frac{f_{1}(\bar{s})}{1-F_{1}(\bar{s})}\right)$, in Eq. $A .2$ is positive in view of the hazard rate inequality (9). Since the term, $E_{1}[s \mid s \geq \bar{s}]-\bar{s}$, is also positive, it follows that $\frac{d p^{o p t}(\bar{s})}{d \bar{s}}<0$. The second part of the statement follows by considering Eq. (11) for $\bar{s}=s^{\min }$ and $\bar{s}=s^{\max }$ 
Proof of Theorem 2, Most of the proof is provided in Section 3.3. The remaining steps are to show that $R_{1}^{L}(\bar{s})$ [cf. Eq. [13] ] has a unique maximum characterized by $p=p^{o p t}(\bar{s})$. To this end, consider:

$$
\frac{d R_{1}^{L}(\tilde{s})}{d \tilde{s}}=\frac{1}{p-E_{0}[\min (s, \tilde{s})]}\left(R_{1}^{L}(\tilde{s})\left(1-F_{0}(\tilde{s})\right)-\left(1-F_{1}(\tilde{s})\right)\right) .
$$

Thus, the first order optimality condition implies:

$$
\frac{R_{1}^{L}(\tilde{s})}{1+r}=\frac{1-F_{1}(\tilde{s})}{1-F_{0}(\tilde{s})}
$$

Plugging this into (13) and rearranging terms yields $p=p^{\text {opt }}(\tilde{s})$. Next note by part (iii) of Lemma 1 that there exists exactly one $\bar{s} \in S$ that satisfies the first order condition.

It remains to show that the unique critical point corresponds to a maximum. To this end, consider the value of the derivative, $\frac{d R_{1}^{L}(\tilde{s})}{d \tilde{s}}$, at the boundaries of region, $\tilde{s}=s^{\min }$ and $\tilde{s}=s^{\max }$. Note that $R_{1}^{L}\left(s^{\min }\right)=\frac{E_{1}[s]-s^{\min }}{p-s^{\min }}>1$ because $p<E_{1}[s]$. Using this inequality, the derivative in (A.3) satisfies $\left.\frac{d R_{1}^{L}(\bar{s})}{d \bar{s}}\right|_{\bar{s}=s^{\min }}>0$. Similarly, note that $R_{1}^{L}\left(s^{\max }\right)=\frac{E_{1}[s]-E_{1}[s]}{p-E_{0}[s]}=0$. Using this in inequality, the derivative in $A .3$ satisfies $\left.\frac{d R_{1}^{L}(\tilde{s})}{d \tilde{s}}\right|_{\tilde{s}=s^{\max }} \leq 0$. These boundary conditions imply that the unique critical point is a maximum of $R_{1}^{L}(\tilde{s})$ over $S$.

Proof of Theorem 3. The analysis in Section 3.3 has characterized the equilibrium as the intersection of the optimality curve, $p^{o p t}(\bar{s})$, and the weakly increasing market clearing curve, $p^{m c}(\bar{s})$. The remaining step is to verify that there is a unique intersection that satisfies $p \in$ $\left(E_{0}[s], E_{1}[s]\right)$. Note that assumption (A1) implies:

$$
p^{o p t}\left(s^{\min }\right)=E_{1}[s]>p^{m c}\left(s^{\min }\right)=n_{1}+s^{\min } .
$$

Note also that:

$$
p^{o p t}\left(s^{\max }\right)=E_{0}[s]<p^{m c}\left(s^{\max }\right)=n_{1}+E_{0}[s] .
$$

Thus, by continuity, $p^{o p t}(\bar{s})$ and $p^{m c}(\bar{s})$ intersect for some interior $\bar{s} \in\left(s^{\min }, s^{\max }\right)$. From part (iii) of Lemma 1, it follows that the intersection is unique and that $p=p^{\text {opt }}(\bar{s}) \in\left(E_{0}[s], E_{1}[s]\right)$.

\section{A.2 General Equilibrium and the Equivalence Result}

This appendix provides the proof for Theorem 1 in Section 3.2 .

Proof of Theorem 1. Part (i). See the proof of Theorem 3.

Parts (ii) and (iii). The proof consists of four steps. The first step notes a number of simplifying observations which must hold in general equilibrium. The second step states the conditions for equilibrium in debt markets in terms of traders' bid and ask prices for debt contracts. The third step characterizes the existence and the essential uniqueness of equilibrium in debt markets corresponding to any asset price $p \in\left[E_{0}[s], E_{1}[s]\right)$. The fourth step combines this analysis with asset market clearing to establish the existence and the essential uniqueness of the general equilibrium, proving part (ii). The analysis in the third and the fourth steps also establish the equivalence result, proving part (iii). 
Step 1 (Simplifying observations). Suppose, without loss of generality, that the asset price satisfies $p \in\left[E_{0}[s], E_{1}[s]\right){ }^{16}$ First, note that problem (5) is linear. Thus, a trader's value function take the form $v_{i} n_{i}$, where $v_{i}$ denotes her return on net worth. Second, traders always have the option of simply investing in cash or the asset, which implies:

$$
v_{i} \geq \max \left(\frac{E_{i}[s]}{p}, 1\right) \text { for each } i
$$

Third, note that the budget constraints are satisfied with equality. Consider the summation of these constraints, which implies: $p+c_{1}+c_{0}=n_{0}+n_{1}$. Since $p<E_{1}[s]$ and $n_{0}+n_{1}>E_{1}[s]$ [cf. assumption (A1)], the previous equality implies $c_{1}+c_{0}>0$. That is, some traders must hold cash in equilibrium. Since cash is not used as collateral, $c_{i}>0$ implies the trader's return on net worth is given by $v_{i}=1$. However, the inequalities in (A.4) along with $p<E_{1}[s]$ imply $v_{1}>1$. It follows that $c_{1}=0$ and $c_{0}>0$. That is, the traders that hold cash are the pessimists, who have return $v_{0}=1$. Finally, it can be checked that debt contracts with promise $\varphi \geq s^{\max }$ are not used in equilibrium. Thus, without loss of generality suppose $\varphi<s^{\max }$.

Step 2 (Conditions for equilibrium in debt markets). Consider the traders' bid prices for debt contracts, given by:

$$
q_{0}^{b i d}(\varphi)=E_{0}[\min (s, \varphi)] \text { and } q_{1}^{b i d}(\varphi)=\frac{E_{1}[\min (s, \varphi)]}{v_{1}} .
$$

Similarly define the trader's ask prices as the price at which trader $i$ would be indifferent to take a negative position at the debt contract. To sell a debt contract, the trader must hold 1 unit of the asset as collateral. Thus, the ask price is found by equating the return on a leveraged investment in the asset with the trader's value per net worth, $v_{i}:{ }^{17}$

$$
\frac{E_{0}[s]-E_{0}[\min (s, \varphi)]}{p-q_{0}^{a s k}(\varphi)}=1 \text { and } \frac{E_{1}[s]-E_{1}[\min (s, \varphi)]}{p-q_{1}^{a s k}(\varphi)}=v_{1} .
$$

It can be checked that $q_{i}^{a s k}(\varphi) \geq q_{i}^{b i d}(\varphi)$ for each $i \in\{0,1\}$. Market clearing for debt contracts imply:

$$
\min _{i} q_{i}^{a s k}(\varphi) \geq q(\varphi) \geq \max _{i} q^{b i d}(\varphi) \text { for each } \varphi .
$$

In addition, a contract, $\varphi$, is traded in positive quantities only if:

$$
q_{i}^{a s k}(\hat{\varphi})=q(\hat{\varphi})=q_{j}^{b i d}(\hat{\varphi}) \text { for some }\{i, j\}=\{0,1\} .
$$

I next claim that

$$
q_{0}^{a s k}(\varphi)>q_{1}^{\text {bid }}(\varphi) \text { for each } \varphi
$$

that is, pessimists' ask prices are always higher than optimists' bid prices. Suppose, to reach a contradiction, that $q_{1}^{\text {bid }}(\tilde{\varphi}) \geq q_{0}^{a s k}(\tilde{\varphi})$ for some $\tilde{\varphi}$. Using the definitions in $(A .5$ ) and $(A .6)$, this

\footnotetext{
${ }^{16}$ The case $p \geq E_{1}[s]$ can be ruled out in view of assumption (A1).

${ }^{17}$ To see this formally, let $\lambda_{i}$ denote the Lagrange multiplier on the collateral constraint (4) for the trader's problem (5). Then, the ask price is the solution to:$$
v_{i} q_{i}^{a s k}(\bar{\varphi})=E_{1}[\min (s, \varphi)]+\lambda_{i} \text { for each } i .
$$

The Lagrange multiplier can be solved from the first order condition for buying a unit of the asset, $E_{i}[s]+\lambda_{i}=v_{i} p$. Plugging this expression into the previous equation gives Eq. A.6.
} 
implies:

$$
\frac{E_{1}[\min (s, \tilde{\varphi})]}{p-\left(E_{0}[s]-E_{0}[\min (s, \tilde{\varphi})]\right)} \geq v_{1}
$$

After using $v_{1} \geq \frac{E_{1}[s]}{p}$ and rearranging terms, this further implies

$$
\frac{E_{1}[s]}{p} \geq \frac{E_{1}[s]-E_{1}[\min (s, \tilde{\varphi})]}{E_{0}[s]-E_{0}[\min (s, \tilde{\varphi})]}
$$

Using the second part of Lemma 1, $\frac{E_{1}[\min (s, \tilde{\varphi})]}{E_{0}[\min (s, \tilde{\varphi})]}$ is strictly increasing over $\tilde{\varphi} \in\left(s^{\min }, s^{\max }\right)$ with maximum value equal to $\frac{E_{1}[s]}{E_{0}[s]}$. Using this observation, it follows that:

$$
\frac{E_{1}[s]-E_{1}[\min (s, \tilde{\varphi})]}{E_{0}[s]-E_{0}[\min (s, \tilde{\varphi})]}>\frac{E_{1}[s]}{E_{0}[s]} \text { for each } \tilde{\varphi}<s^{\max } .
$$

Combining the last two equations yields a contradiction since $p \geq E_{0}[s]$.

In view of the claim in $A .7$, there is no contract with positive trade in which optimists buy and pessimists sell. Put differently $\hat{\mu}_{1}^{+}=\hat{\mu}_{1}^{-}=0$, and the equilibrium in debt contracts can be rewritten as:

$$
\left\{\begin{array}{c}
q(\hat{\varphi})=q_{0}^{b i d}(\hat{\varphi})=q_{1}^{a s k}(\hat{\varphi}), \text { for each } \hat{\varphi} \text { with positive trade } \\
q(\varphi) \in\left[\max _{i} q^{b i d}(\varphi), \min _{i} q^{a s k}(\varphi)\right], \text { for each } \varphi
\end{array}\right.
$$

Step 3 (Characterizing the essentially unique equilibrium in debt markets). I next calculate optimists' value function and borrowing portfolio in equilibrium, $v_{1}$ and $\hat{\mu}_{1}^{-}$. I assume optimists face quasi-equilibrium debt prices:

$$
\tilde{q}(\varphi)=q_{0}^{b i d}(\varphi)=E_{0}[\min (s, \varphi)]
$$

This is without loss of generality for the purposes of calculating $v_{1}$ and $\hat{\mu}_{1}^{-}$, even though the equilibrium prices might be different than the quasi-equilibrium prices ${ }^{18}$ Given the quasi-equilibrium prices, optimists' solve the following optimization problem:

$$
\begin{array}{ccc}
v_{1} n_{1}=\max _{a_{1} \geq 0, \mu_{1}^{-}} & a_{1} E_{1}[s]-\int_{\varphi \in \mathbb{R}_{+}} E_{1}[\min (s, \varphi)] d \mu_{1}^{-}, & \\
\text {s.t. } & p a_{1}-\int_{\varphi \in \mathbb{R}_{+}} E_{0}[\min (s, \varphi)] d \mu_{1}^{-}=n_{1} & \text { [budget constraint], } \\
& \int_{\varphi \in \mathbb{R}_{+}} d \mu_{1}^{-} \leq a_{1} . & \text { [collateral constraint]. }
\end{array}
$$

Here, the first line uses Fubini's Theorem to replace $\int_{\varphi \in \mathbb{R}_{+}} E_{1}[\min (s, \varphi)] d \mu_{1}^{-}=$ $E_{1}\left[\int_{\varphi \in \mathbb{R}_{+}} \min (s, \varphi) d \mu_{1}^{-}\right]$. Since $p<E_{1}[s]$, the collateral constraint binds. Using the envelope theorem, $v_{1}$ corresponds to the Lagrange multiplier on the budget constraint. Let $\lambda_{1}$ denote the Lagrange multiplier for the collateral constraint. The first order conditions for $a_{1}$ and $\mu_{1}^{-}$are

\footnotetext{
${ }^{18}$ To see this, note that $\tilde{q}(\varphi) \leq q(\varphi)$ for each $\varphi$. Thus, optimists' equilibrium problem is different than their quasi-equilibrium problem only if there is a contract $\tilde{\varphi}$ such that $\tilde{q}(\tilde{\varphi})=q_{0}^{\text {bid }}(\tilde{\varphi})<q(\tilde{\varphi})$. In addition, optimists' value function is different only if there is positive trade in this contract, which requires $q(\tilde{\varphi})=q_{1}^{\text {bid }}(\tilde{\varphi})=q_{0}^{\text {ask }}(\tilde{\varphi})$. This provides a contradiction to the claim in A.7.
} 
respectively given by:

$$
\begin{aligned}
& E_{1}[s]+\lambda_{1}=v_{1} p, \text { and } \\
& v_{1} E_{0}[\min (s, \varphi)] \leq E_{1}[\min (s, \varphi)]+\lambda_{1}, \text { with equality only if } \varphi \in \operatorname{supp}\left(\mu_{1}^{-}\right) .
\end{aligned}
$$

Combining these first order conditions yields:

$$
R_{1}^{L}(\varphi)=\frac{E_{1}[s]-E_{1}[\min (s, \varphi)]}{p-E_{0}[\min (s, \varphi)]} \leq v_{1} \text {, with equality only if } \varphi \in \operatorname{supp}\left(\mu_{1}^{-}\right) .
$$

Recall from Appendix A.1 that $R_{1}^{L}(\varphi)$ has a unique maximum characterized by $p=p^{\text {opt }}(\hat{\varphi})$. It follows that there is a unique solution to the first order condition A.11, $\hat{\varphi}$. Thus, the optimists' borrowing portfolio, $\hat{\mu}_{1}^{-}$, is a Dirac measure that puts weight only at one contract $\hat{\varphi} \in \mathbb{R}_{+}$. In addition, optimists' value per net worth is given by $v_{1}=R_{1}^{L}(\hat{\varphi})$.

Combining this analysis with the characterization in $A .8$, it follows that the equilibria in the debt markets are characterized by the following conditions:

$$
\begin{aligned}
q(\hat{\varphi}) & =E_{0}[\min (s, \hat{\varphi})], \text { where } \hat{\varphi} \text { is the solution to } p=p^{\text {opt }}(\hat{\varphi}), \\
\text { and } q(\varphi) & \in\left[\max _{i} q^{b i d}(\varphi), \min _{i} q^{a s k}(\varphi)\right] \text { for each } \varphi \neq \hat{\varphi}
\end{aligned}
$$

I next claim that this set is non-empty, that is, there exists an equilibrium in debt markets. In particular, consider the following (bid) prices: $q(\varphi)=\max \left(q_{0}^{\text {bid }}(\varphi), q_{1}^{\text {bid }}(\varphi)\right)$. In view of (A.12), these prices constitute an equilibrium if and only if $q_{0}^{b i d}(\hat{\varphi})=E_{0}[\min (s, \hat{\varphi})]>q_{1}^{b i d}(\hat{\varphi})$. This in turn is the case because $q_{0}^{\text {bid }}(\hat{\varphi})=q_{1}^{\text {ask }}(\hat{\varphi})>q_{1}^{\text {bid }}(\hat{\varphi})$.

This analysis also establishes that the equilibrium in debt markets is essentially unique in the sense that the value per net worth, $v_{1}$, the traded contract, $\hat{\varphi}$, and the price of the traded contract, $E_{0}[\min (s, \hat{\varphi})]$, are uniquely determined. However, the price of non-traded debt contracts are not uniquely determined and their prices can typically take a continuum of values.

Step 4 (Characterizing the essentially unique general equilibrium). Next consider asset market clearing. Since the collateral constraint in $A .10$ binds, optimists sell $\hat{a}_{1}$ units of the traded contract, $\hat{\varphi}$. Plugging this into the budget constraint in $A .10$ implies: $p \hat{a}_{1}-E_{0}[\min (s, \hat{\varphi})] \hat{a}_{1}=$ $n_{1}$. Since only optimists demand the asset, market clearing requires $\hat{a}_{1}=1$. Plugging this into the previous equation gives the market clearing relation $p=p^{m c}(\hat{\varphi})$ [cf. Eq. (16)]. It follows that a traded contract and price pair, $(\hat{\varphi}, \hat{p})$, corresponds to a general equilibrium if and only if it solves $\hat{p}=p^{o p t}(\hat{\varphi})=p^{m c}(\hat{\varphi})$. In view of Theorem 3, there is a unique pair, $(\hat{\varphi}, \hat{p})$, that solves these equations. It follows that there exists an essentially unique general equilibrium which is equivalent to the principal agent equilibrium.

\section{A.3 Comparative Statics with Simple Debt Contracts}

This appendix provides proofs for the theorems in Section 4 .

Proof of Theorem 4. Define the function $g: S \rightarrow R$ with $g(\bar{s})=\tilde{E}_{1}[s \mid s \geq \bar{s}]-E_{1}[s \mid s \geq \bar{s}]$. Note that $g\left(s^{\min }\right)=0$ since $\tilde{E}_{1}[s]=E_{1}[s]$, and also that $g\left(s^{\max }\right)=0$. I claim that:

$$
g(\bar{s}) \geq 0 \text { for all } \bar{s} \in\left(s^{\min }, s^{\max }\right) .
$$


The comparative statics for $p$ and $\bar{s}^{*}$ then follow by the argument provided after Theorem 4. For the comparative statics of the margin, substitute Eq. (16) into (17) to get $m=n_{1} / p$. It follows that the margin decreases because $p$ increases and $n_{1}$ is unchanged. If $\tilde{F}_{1}$ satisfies condition (18) strictly, then the inequality in $A .13$ holds strictly and the comparative statics for $p, \bar{s}^{*}$ and $m$ also become strict.

The remaining step is the proof of claim $A .13$. I start by calculating $g^{\prime}(\bar{s})$. Consider first the derivative of the conditional valuation:

$$
\frac{d \tilde{E}_{1}[s \mid s \geq \bar{s}]}{d \bar{s}}=\frac{\tilde{f}_{1}(\bar{s})}{1-\tilde{F}_{1}(\bar{s})}\left(\tilde{E}_{1}[s \mid s \geq \bar{s}]-\bar{s}\right) .
$$

This expression further implies:

$$
\begin{aligned}
g^{\prime}(\bar{s}) & =\frac{\tilde{f}_{1}(\bar{s})}{1-\tilde{F}_{1}(\bar{s})}\left(\tilde{E}_{1}[s \mid s \geq \bar{s}]-\bar{s}\right)-\frac{f_{1}(\bar{s})}{1-F_{1}(\bar{s})}\left(E_{1}[s \mid s \geq \bar{s}]-\bar{s}\right) \\
& =\left(\frac{\tilde{f}_{1}(\bar{s})}{1-\tilde{F}_{1}(\bar{s})}-\frac{f_{1}(\bar{s})}{1-F_{1}(\bar{s})}\right)\left(\tilde{E}_{1}[s \mid s \geq \bar{s}]-\bar{s}\right)+\frac{f_{1}(\bar{s})}{1-F_{1}(\bar{s})} g(\bar{s})
\end{aligned}
$$

I next prove the claim in $A .13$ in two steps. As the first step, consider $g(\bar{s})$ over the range $\bar{s} \in\left[s^{\mathrm{min}}, s^{R}\right]$. Note that $\frac{f_{1}(\bar{s})}{1-\tilde{F}_{1}(\bar{s})} \geq \frac{f_{1}(\bar{s})}{1-F_{1}(\bar{s})}$ for $s<s^{R}$. Thus, Eq. A.14 constitutes a differential equation of the form $g^{\prime}(\bar{s})=A(\bar{s})+B(\bar{s}) g(\bar{s})$ where $A(\bar{s}) \geq 0$ and $B(\bar{s})>0$, with initial condition $g\left(s^{\min }\right)=0$. It follows that $g(\bar{s}) \geq 0$ for each $\bar{s} \in\left[s^{\min }, s^{R}\right]$, proving the claim over this range.

As the second step, consider $g(\bar{s})$ over the range $\bar{s} \in\left[s^{R}, s^{\max }\right]$. Note that $\frac{\tilde{f}_{1}(\bar{s})}{1-\tilde{F}_{1}(\bar{s})} \leq \frac{f_{1}(\bar{s})}{1-F_{1}(\bar{s})}$ for $s>s^{R}$. Note also that $\tilde{E}_{1}[s \mid s \geq \bar{s}]-\bar{s} \geq 0$. Using these inequalities, Eq. A.14 implies:

$$
g^{\prime}(\bar{s}) \leq \frac{\tilde{f}_{1}(\bar{s})}{1-\tilde{F}_{1}(\bar{s})} g(\bar{s}) \text { for each } \bar{s} \in\left[s^{R}, s^{\max }\right) .
$$

Next suppose, to reach a contradiction, that there exists $\hat{s}<s^{\max }$ such that $g(\hat{s})<0$. Define $\hat{s}^{\max }=\sup \left\{s \in\left[\hat{s}, s^{\max }\right) \mid g(s) \leq g(\hat{s})\right\}$. Note that $g\left(\hat{s}^{\max }\right)=g(\hat{s})<0$ by the continuity of $g(\cdot)$. Note also that $\hat{s}^{\max }<s^{\max }$ since $g\left(s^{\max }\right)=0$. Then, the inequality in $A .15$ applies for $\hat{s}^{\max }$ and implies $g^{\prime}\left(\hat{s}^{\max }\right) \leq \frac{\tilde{f}_{1}(\hat{s})}{1-\tilde{F}_{1}(\hat{s})} g\left(\hat{s}^{\max }\right)<0$. This further implies that there exists $s \in\left(\hat{s}^{\max }, s^{\max }\right)$ such that $g(s)<g\left(\hat{s}^{\max }\right)=g(\hat{s})$, which contradicts the definition of $\hat{s}^{\max }$. It follows that $g(\bar{s}) \geq 0$ for each $\bar{s} \in\left[s^{R}, s^{\max }\right]$, proving the claim also over this range.

I next consider the proof of Theorem 5. The proof uses the following lemma.

Lemma 2. Consider two probability distributions $F_{1}$ and $F_{0}$ that satisfy assumption (A2).

(i) Suppose optimists become weakly more optimistic, i.e., consider their beliefs are changed to $\tilde{F}_{1}$ that satisfies the weak hazard rate inequality in $(19)$. Then, the optimality curve shifts up pointwise, that is:

$$
p^{o p t}\left(\bar{s} ; \tilde{F}_{1}\right) \geq p^{o p t}\left(\bar{s} ; F_{1}\right) \text { for each } \bar{s} \in S .
$$

The market clearing curve is unchanged.

(ii) Suppose pessimists become weakly more pessimistic, i.e., consider their beliefs are changed to $\tilde{F}_{0}$ that satisfies the weak hazard rate inequality in (19). Then, the optimality curve shifts down 
pointwise, that is:

$$
p^{o p t}\left(\bar{s} ; \tilde{F}_{0}\right) \leq p^{o p t}\left(\bar{s} ; F_{0}\right) \text { for each } \bar{s} \in S .
$$

Moreover, the market clearing curve also shifts down, that is:

$$
p^{m c}\left(\bar{s} ; \tilde{F}_{0}\right) \leq p^{m c}\left(\bar{s} ; F_{0}\right) \text { for each } \bar{s} \in S \text {. }
$$

Proof of Lemma 2, Part (i). In view of Eq. (12), it suffices to show that

$$
\tilde{E}_{1}[s \mid s \geq \bar{s}] \geq E_{1}[s \mid s \geq \bar{s}] \text { for each } \bar{s} \in S .
$$

Note that $\frac{\tilde{f}_{1}(\bar{s})}{1-\tilde{F}_{1}(\bar{s})} \leq \frac{f_{1}(\bar{s})}{1-F_{1}(\bar{s})}$ for each $\bar{s} \in S$. Thus, the previous displayed inequality follows from the argument in (the second step of) the proof of Theorem 4 .

Part (ii). To show (A.16), define the function $h: S \rightarrow R$ with

$$
h(\bar{s})=p^{o p t}\left(\bar{s} ; F_{0}\right)-p^{o p t}\left(\bar{s} ; \tilde{F}_{0}\right) .
$$

Note that $A .16$ is equivalent to showing that $h(\bar{s}) \geq 0$ for each $\bar{s} \in S$. I will prove the stronger claim that $h(\bar{s})$ is weakly increasing over $S$. This claim implies that $h(\bar{s}) \geq 0$ because $h\left(s^{\min }\right)=0$. To prove that $h(\bar{s})$ is weakly increasing, consider the derivative of $h(\bar{s})$. After using Eq. A.2 and rearranging terms, this derivative can be written as:

$$
\frac{d h(\bar{s})}{d \bar{s}}=\left(\begin{array}{c}
\tilde{f}_{0}(\bar{s})-f_{0}(\bar{s}) \\
-\frac{f_{1}(\bar{s})}{1-F_{1}(\bar{s})}\left(F_{0}(\bar{s})-\tilde{F}_{0}(\bar{s})\right)
\end{array}\right)\left(E_{1}[s \mid s \geq \bar{s}]-\bar{s}\right) .
$$

Next note that $\frac{\tilde{f}_{0}(\bar{s})}{1-\tilde{F}_{0}(\bar{s})} \geq \frac{f_{0}(\bar{s})}{1-F_{0}(\bar{s})}$, which also implies $\frac{\tilde{f}_{0}(\bar{s})-f_{0}(\bar{s})}{F_{0}(\bar{s})-\tilde{F}_{0}(\bar{s})} \geq \frac{f_{0}(\bar{s})}{1-F_{0}(\bar{s})}$. Combining this inequality with $\frac{f_{0}(\bar{s})}{1-F_{0}(\bar{s})} \geq \frac{f_{1}(\bar{s})}{1-F_{1}(\bar{s})}$ implies:

$$
\frac{\tilde{f}_{0}(\bar{s})-f_{0}(\bar{s})}{F_{0}(\bar{s})-\tilde{F}_{0}(\bar{s})} \geq \frac{f_{1}(\bar{s})}{1-F_{1}(\bar{s})} .
$$

Using this inequality in Eq. A.18 shows that $\frac{d h(\bar{s})}{d \bar{s}} \geq 0$, which completes the proof of A.16.

To show $A .17$, recall from Lemma 1 that $\frac{E_{1}[\min (s, \bar{s})]}{E_{0}[\min (s, \bar{s})]}$ is increasing in $\bar{s}$. Taking $F_{0}$ in place of $F_{1}$ and $\tilde{F}_{0}$ in place of $F_{0}$, this implies $\frac{E_{0}[\min (s, \bar{s})]}{\tilde{E}_{0}[\min (s, \bar{s})]}$ is increasing in $\bar{s}$. This in turn implies:

$$
E_{0}[\min (s, \bar{s})] \geq \tilde{E}_{0}[\min (s, \bar{s})] \text { for each } \bar{s} \in \mathcal{S} .
$$

Using this inequality in Eq. 16 proves $A .17$.

Proof of Theorem 5. Part (i). The fact that the conditions in (19) are satisfied with equality over $\left(s^{\mathrm{min}}, \bar{s}^{*}\right)$ implies $F_{0}$ and $F_{1}$ are unchanged over this range. From Eq. (16), it follows that $p^{m c}(\bar{s})$ is also unchanged over this range. On the other hand, I claim that $p^{o p t}(\bar{s})$ shifts up over this range. Since $p^{o p t}(\cdot)$ is decreasing and $p^{m c}(\cdot)$ is increasing, this claim implies that the new intersection point is for a greater $\bar{s}^{*}$ and a greater $p$. It follows that $p$ and $\bar{s}^{*}$ increase. This also implies that the margin $m=n_{1} / p$ decreases. 
Thus, it remains to prove the claim that $p^{o p t}(\bar{s})$ shifts up over the range, $\left(s^{\min }, \bar{s}^{*}\right)$. To this end, first note that part (i) of Lemma 2 implies:

$$
p^{o p t}\left(\bar{s} ; F_{0}, \tilde{F}_{1}\right) \geq p^{o p t}\left(\bar{s} ; F_{0}, F_{1}\right) \text { for each } \bar{s} \in S \text {. }
$$

That is, ignoring the change in $F_{0}$, the optimality curve shifts up. Next recall that $F_{0}$ is unchanged over $\left(s^{\min }, \bar{s}^{*}\right)$. By Eq. (11), this implies:

$$
p^{o p t}\left(\bar{s} ; \tilde{F}_{0}, \tilde{F}_{1}\right)=p^{o p t}\left(\bar{s} ; F_{0}, \tilde{F}_{1}\right) \text { for each } \bar{s} \in\left(s^{\min }, \bar{s}^{*}\right) \text {. }
$$

That is, the change in $F_{0}$ [which is concentrated on the region $\left(\bar{s}^{*}, s^{\max }\right)$ ] does not affect the optimality curve over the region $\left(s^{\min }, \bar{s}^{*}\right)$. Combining Eq. A.20 with the inequality in A.19 proves that $p^{o p t}(\bar{s})$ shifts up for $\bar{s} \in\left(s^{\min }, \bar{s}^{*}\right)$.

Part (ii). Parts (i) and (ii) of Lemma 2 imply that $p^{m c}(\bar{s})$ shifts down over the entire range $S$. I also claim that $p^{o p t}(\bar{s})$ shifts down over the range $\left(\bar{s}^{*}, s^{\max }\right)$. Since $p^{\text {opt }}(\cdot)$ is decreasing and $p^{m c}(\cdot)$ is increasing, this claim implies that the new intersection point is for a lower price $p$. It also follows that the margin $m=n_{1} / p$ increases.

Thus, it remains to prove the claim that $p^{\text {opt }}(\bar{s})$ shifts down over the range $\left(\bar{s}^{*}, s^{\text {max }}\right)$. To this end, first note that part (ii) of Lemma 2 implies:

$$
p^{o p t}\left(\bar{s} ; \tilde{F}_{0}, F_{1}\right) \leq p^{o p t}\left(\bar{s} ; F_{0}, F_{1}\right) \text { for each } \bar{s} \in S .
$$

That is, ignoring the change in $F_{1}$, the optimality curve shifts down. Next recall that by assumption $\frac{f_{0}}{1-F_{0}}$ is unchanged over $\left(\bar{s}^{*}, s^{\max }\right)$. By Eq. (11), this implies:

$$
p^{o p t}\left(\bar{s} ; \tilde{F}_{0}, \tilde{F}_{1}\right)=p^{o p t}\left(\bar{s} ; \tilde{F}_{0}, F_{1}\right) \text { for each } \bar{s} \in\left(\bar{s}^{*}, s^{\max }\right) .
$$

That is, the change in $F_{1}$ [which is concentrated on the region $\left(s^{\min }, \bar{s}^{*}\right)$ ] does not affect the optimality curve over the region $\left(\bar{s}^{*}, s^{\max }\right)$. Combining Eq. A.22 with the inequality in A.21) proves that $p^{o p t}(\bar{s})$ shifts down for $\bar{s} \in\left(\bar{s}^{*}, s^{\max }\right)$.

\section{A.4 Equilibrium with Short Contracts}

This appendix completes the characterization of the equilibrium analyzed in Section 3.3 .

Proof of Theorem 6. I first show that there is unique solution to $p=p^{o p t, S}(\gamma)$. With some algebra, the derivative of $p^{\text {opt,S }}(\gamma)$ in Eq. 25) can be calculated as:

$$
\frac{d p^{o p t, S}(\gamma)}{d \gamma}=-\frac{E_{1}[s]\left(\int_{s^{\min }}^{\gamma} s d F_{1}\right)}{\left(1-F_{1}(\gamma)+F_{0}(\gamma) \frac{\int_{s \min }^{\gamma} s d F_{1}}{\int_{s^{\min }}^{\gamma} s d F_{0}}\right)^{2}}\left[\frac{f_{1}(\gamma)}{\int_{s^{\min }}^{\gamma} s d F_{1}}-\frac{f_{0}(\gamma)}{\int_{s^{\min }}^{\gamma} s d F_{0}}\right]\left[\frac{F_{0}(\gamma) \gamma}{\int_{s^{\min }}^{\gamma} s d F_{0}}-1\right]
$$

It can be checked that the last bracketed term is positive. The second to last bracketed term is also positive in view of assumption $\left(\mathrm{A} 2^{S}\right)$. This implies $\frac{d p^{o p t, S}(\gamma)}{d \gamma}<0$. Note also that $p^{o p t, S}\left(s^{\min }\right)=$ $E_{1}[s]$ and $p^{o p t, S}\left(s^{\max }\right)=E_{0}[s]$. Since $p \in\left(E_{0}[s], E_{1}[s]\right)$, it follows that there is a unique solution to $p=p^{o p t, S}(\gamma)$ over the range $\gamma \in\left[s^{\min }, s^{\max }\right]$.

I next show that the unique solution to $p=p^{o p t, S}(\gamma)$ corresponds to the solution to problem 
(24). Substituting $x_{0}$ from the budget constraint, this problem can be written as:

$$
\max _{\gamma} R_{1}^{S}(\gamma) \equiv \frac{\gamma-E_{0}[\min (\gamma, s)]}{\gamma-\frac{1}{\frac{E_{1}[s]}{p}} E_{1}[\min (\gamma, s)]}
$$

This expression is the expected return of pessimists who sell one unit of short contract with cash collateral $\gamma$. The derivative of $R_{1}^{S}(\gamma)$ can be calculated as:

$$
\frac{d R_{1}^{S}(\gamma)}{d \gamma}=R_{1}^{S}(\gamma)\left(\frac{F_{0}(\gamma)}{\gamma-E_{0}[\min (\gamma, s)]}-\frac{1-\frac{1}{\frac{E_{1}[s]}{p}}\left(1-F_{1}(\gamma)\right)}{\gamma-\frac{1}{\frac{E_{1}[s]}{p}} E_{1}[\min (\gamma, s)]}\right)
$$

Setting this expression to zero and rearranging terms implies the first order condition $p=$ $p^{o p t, S}(\gamma)$. Since there is a unique solution to $p=p^{o p t, S}(\gamma)$, it follows that there is a unique maximum $R_{1}^{S}(\gamma)$ characterized by the first order condition.

Asset market clearing and characterization of equilibrium. Eq. (22) implies $m^{S}=$ $\frac{\gamma-q(\gamma)}{p}$. Substituting for $q(\gamma)$ from Eq. 23) gives

$$
m^{S}(\gamma, p)=\frac{1}{p}\left(\gamma-p \frac{E_{1}[\min (\gamma, s)]}{E_{1}[s]}\right) .
$$

Using this expression, Eq. (26) can be written as:

$$
n_{1}=p\left(1+\frac{n_{0}}{\gamma \frac{E_{1}[s]}{E_{1}[\min (\gamma, s)]}-p}\right) .
$$

Note that the right hand side is strictly increasing in $p$. Consequently, for each $\gamma \in\left[s^{\min }, s^{\max }\right]$, there is a unique solution to this equation denoted by $p^{m c, S}(\gamma)$. Moreover, $p^{m c, S}(\gamma)$ is increasing because the right hand side is strictly decreasing in $\gamma$. It can also be checked that $p^{m c, S}\left(s^{\min }\right)<$ $E_{1}[s]$ and that $p^{m c, S}\left(s^{\max }\right)>E_{0}[s]$ [in view of assumption $\left(\mathrm{A} 1^{\mathrm{S}}\right)$ ]. Consequently, $p^{\text {opt }, S}(\gamma)$ and $p^{m c, S}(\gamma)$ intersect at some $\gamma \in\left(s^{\min }, s^{\max }\right)$ and $p \in\left(E_{0}[s], E_{1}[s]\right)$. This completes the characterization of equilibrium.

\section{A.5 Equilibrium with Simple Short and Debt Contracts}

Sections 3 and 5 considered the equilibrium with either simple debt contracts or simple short contracts in isolation. This appendix presents a model that features both types of contracts, and shows that the asymmetric disciplining result continues to apply in this setting.

Consider a variant of the general equilibrium defined in Section 2 with the contract space $\mathcal{B}^{T}=\mathcal{B}^{D} \cup \mathcal{B}^{S}$, where $\mathcal{B}^{D}$ and $\mathcal{B}^{S}$ are respectively the set of simple debt and short contracts defined in Eqs. (7) and (21). To keep the analysis tractable, I also assume that only a fraction $\gamma^{S} \in[0,1]$ of traders can sell short contracts, while only a fraction $\gamma^{D} \in[0,1]$ can sell debt contracts. That is, only a fraction of traders can short sell the asset or leverage their investments in the asset. These assumptions are not unreasonable because short selling in financial markets (and to some extent, leverage) is confined to a small fraction of investors. Assume also that the parameters $\left(n_{0}, n_{1}, \gamma^{S}, \gamma^{D}\right)$ are such that the equilibrium price is interior, $p \in\left(E_{0}[s], E_{1}[s]\right)$ [which will be verified after the characterization]. 
Under these assumptions, there exists an equilibrium of the following form. The fraction, $\gamma^{D}$, of optimists leverage their investments in the asset using a loan with riskiness $\bar{s} \in S$. The debt contracts they sell are bought by the fraction, $1-\gamma^{S}$, of pessimists who are unable to short sell. On the other hand, the fraction, $\gamma^{S}$, of pessimists short sell the asset using cash collateral $\gamma \in S$. The short contracts they sell are bought by the fraction, $1-\gamma^{D}$, of optimists who are unable to leverage. To see the intuition for this matching, note that pessimists that are able to short sell require a greater interest rate to part with their endowment than those who are unable to short sell. This is because they have a greater expected rate of return on their endowment (in view of their ability to short sell). Consequently, optimists borrow cash from the latter type of pessimists. A similar reasoning shows that pessimists borrow the asset from optimists who are unable to leverage.

An equilibrium can then be represented by a triple, $(p, \bar{s}, \gamma)$. Moreover, the optimal debt and short contracts are characterized by the same analysis in Sections 3 and 5 . In particular, the riskiness of the optimal loan, $\bar{s}$, is characterized as the solution to $p=p^{o p t}(\bar{s})$ [cf. Eq. (11)]. Similarly, the cash-collateral of the optimal short contract is characterized as the solution to $p=p^{m c}(\bar{s})$. Assume that beliefs satisfy assumption (MLRP) of Section 6, which ensures that the optimal debt and short contracts are uniquely determined. The remaining step is asset market clearing, which can be written as:

$$
\frac{n_{1}}{p}\left[\gamma^{D} \frac{1}{m(\bar{s}, p)}+1-\gamma^{D}\right]=1+\gamma^{S} \frac{n_{0}}{p}\left[\frac{1}{m^{S}(\gamma, p)}\right] \frac{E_{1}[\min (\gamma, s)]}{E_{1}[s]} .
$$

Here, $m(\bar{s}, p)$ denotes the loan margin [cf. Eq. [17)] and $m^{S}(\gamma, p)$ denotes the short margin [cf. Eq. A.23]. The left hand side of Eq. A.25 is the total demand for the asset which consists of demand by the fraction, $\gamma^{D}$, of optimists who leverage their investments and the fraction, $1-\gamma^{D}$, of optimists who are unable to leverage. The right hand side is the total supply of the asset which consists of the physical supply of 1 unit and the short contracts sold by the fraction, $\gamma^{S}$, of pessimists who are able to short sell.

Eq. A.25 defines a third (market clearing) equation between the asset price $p$ and the contracts $(\bar{s}, \gamma)$. The equilibrium triple, $(p, \bar{s}, \gamma)$, is characterized by the solution to this equation along with the optimality conditions $p=p^{o p t}(\bar{s})=p^{o p t, S}(\gamma)$. To illustrate the asymmetric disciplining result, suppose the belief disagreements shift to upside states (while keeping $E_{1}[s]$ and $E_{0}[s]$ constant). By the analysis in Section 4, this induces optimists to leverage using loans with a smaller riskiness, $\bar{s}$, and a lower loan margin, $m$. By the analysis in Section 5 , this also induces pessimists to short sell using a larger cash-collateral, $\gamma$, and a higher short margin, $m^{S}$. In view of these observations, the market clearing relation $A .25$ implies that (keeping $p$ constant) the demand for the asset increases while the effective supply of the asset decreases. This in turn leads to a higher equilibrium price. Conversely, if the belief disagreements shift to downside states, then the loan margin increases, the short margin decreases, and the asset price decreases.

\section{A.6 Equilibrium with Richer Contracts}

This appendix presents the proofs of the results in Section 6. It also presents an example to illustrate that richer contracts have an ambiguous effect on the asset price.

Proof of Theorem 7, Given any portfolio, $\left(a_{i}, c_{i}, \mu_{i}^{+}, \mu_{i}^{-}\right)$, define:

$$
z_{i}(s) \equiv \int_{\mathcal{B}^{T}} \min (\boldsymbol{\varphi}(s), \alpha s+\gamma) d \mu_{i}^{+}-\int_{\mathcal{B}^{T}} \min (\boldsymbol{\varphi}(s), \alpha s+\gamma) d \mu_{i}^{-} \text {for each } s \in S,
$$


as the corresponding $\mathrm{AD}$ positions for type $i$ traders. Given the prices in (29), the budget constraint (3) can be written as $a_{i} p^{*}+c_{i}+\int_{S} z_{i}(s) q^{A D}(s) d s \leq n_{i}$. Note also that the collateral constraint implies that $a_{i} s+c_{i}-z_{i}(s) \geq 0$ a.e. $s$. Hence, a portfolio is feasible in problem (5) only if the corresponding portfolio, $\left(a_{i}, c_{i}, z_{i}\right)$, is feasible in problem (27). In addition, note also that the objective values of the two problems are identical because Eq. A.26 implies:

$$
\begin{gathered}
a_{i} E_{i}[s]+c_{i}+E_{i}\left[\int_{\mathcal{B}^{T}} \min (\varphi(s), \alpha s+\gamma) d \mu_{i}^{+}\right] \\
-E_{i}\left[\int_{\mathcal{B}^{T}} \min (\varphi(s), \alpha s+\gamma) d \mu_{i}^{-}\right]
\end{gathered}=\int_{S}\left(a_{i} s+c_{i}+z_{i}(s)\right) d F_{i} .
$$

Next note that the constructed portfolios, $\left(\hat{a}_{i}, \hat{c}_{i}, \hat{\mu}_{i}^{+}, \hat{\mu}_{i}^{-}\right)$, are feasible for traders given the constructed prices, $(\hat{p},[\hat{q}(\cdot)])$ (since the contract space is sufficiently rich). Moreover, the portfolio, $\left(\hat{a}_{i}, \hat{c}_{i}, \hat{\mu}_{i}^{+}, \hat{\mu}_{i}^{-}\right)$, yields type $i$ traders the same utility as the $\mathrm{AD}$ portfolio, $\left(a_{i}^{*}, c_{i}^{*}, z_{i}^{*}\right)$. I next claim that the portfolio, $\left(\hat{a}_{i}, \hat{c}_{i}, \hat{\mu}_{i}^{+}, \hat{\mu}_{i}^{-}\right)$, is also optimal for these traders, i.e., it solves problem (5) with prices $(\hat{p},[\hat{q}(\cdot)])$. Suppose, to reach a contradiction, that there is an alternative portfolio, $\left(\tilde{a}_{i}, \tilde{c}_{i}, \tilde{\mu}_{i}^{+}, \tilde{\mu}_{i}^{-}\right)$, that results in a strictly higher value. Construct the effective AD positions, $\tilde{z}_{i}(s)$, as above. Note that the alternative AD portfolio, $\left(\tilde{a}_{i}, \tilde{c}_{i}, \tilde{z}_{i}\right)$, is feasible for problem (5). It also results in a strictly higher value for type $i$ traders than the portfolio, $\left(a_{i}^{*}, c_{i}^{*}, z_{i}^{*}\right)$. This implies that $\left(a_{i}^{*}, c_{i}^{*}, z_{i}^{*}\right)$ is not optimal for problem (5), which yields a contradiction. It follows that the constructed portfolios and prices, $\left[\left(\hat{a}_{i}, \hat{c}_{i}, \hat{\mu}_{i}^{+}, \hat{\mu}_{i}^{-}\right),(\hat{p},[\hat{q}(\cdot)])\right]$, constitute a general equilibrium.

Proof of Theorem 8, First consider prices and traders' positions in AD securities. Assumption $\left(\mathrm{A} 1^{R}\right)$ ensures that, in equilibrium, both the asset and cash are held in positive quantities. Thus, by no-arbitrage, their prices satisfy:

$$
p=\int_{S} s q^{A D}(s) d s \text { and } 1=\int_{S} q^{A D}(s) d s .
$$

Given these pricing relations, type $i$ traders' problem (27) can be simplified as:

$$
\begin{array}{ll} 
& \max _{z_{i}^{\text {net }}: S \rightarrow \mathbb{R}_{+}} \int_{S} z_{i}^{\text {net }}(s) d s \\
\text { s.t. } & \int_{S} z_{i}^{\text {net }}(s) q^{A D}(s) d s=n_{1} \text { and } z_{i}^{\text {net }}(s) \geq 0 \text { a.e. on } S,
\end{array}
$$

where $z_{i}^{\text {net }}(s)=a_{i} s+c_{i}+z_{i}(s)$ denotes consumption in state $s$. Let $R_{i}$ denote the Lagrange multiplier on the budget constraint and consider the first order condition:

$$
z_{i}^{\text {net }}(s)=\left\{\begin{array}{cl}
\infty, & \text { if } \frac{f_{i}(s)}{q^{A D}(s)}>R_{i} \\
\mathbb{R}_{+}, & \text {if } \frac{f_{i}(s)}{q^{A D}(s)}=R_{i}, \text { a.e. on } S . \\
0, & \text { if } \frac{f_{i}(s)}{q^{A D}(s)}<R_{i}
\end{array}\right.
$$

Market clearing in $\mathrm{AD}$ securities then requires $q^{A D}(s)=\max \left(\frac{f_{0}(s)}{R_{0}}, \frac{f_{1}(s)}{R_{1}}\right)$ a.e. on $S$. In view of assumption (MLRP), this implies that the AD security prices are given by Eq. (31) and traders' positions in these securities are given by Eq. (30).

Next consider the solution to Eqs. (31) - 34). Fix some $\bar{s} \in\left[s^{\min }, s^{\max }\right]$ and note that $\frac{R_{0}}{R_{1}}=\frac{f_{0}(\bar{s})}{f_{1}(\bar{s})}$. Using this expression, Eq. (33) characterizes pessimists' rate of return: 


$$
R_{0}(\bar{s})=F_{0}(\bar{s})+\frac{f_{0}(\bar{s})}{f_{1}(\bar{s})}\left(1-F_{1}(\bar{s})\right) .
$$

In view of assumption (MLRP), $R_{0}(\bar{s})$ is a strictly decreasing function that satisfies $R_{0}\left(s^{\min }\right)=$ $\frac{f_{0}\left(s^{\min }\right)}{f_{1}\left(s^{\min }\right)}$ and $R_{0}\left(s^{\max }\right)=1$. Using these expressions, Eq. 32 can be written as:

$$
p=p^{o p t, A D}(\bar{s}) \equiv \frac{\int_{s^{\min }}^{\bar{s}} s f_{0}(s) d s+\frac{f_{0}(\bar{s})}{f_{1}(\bar{s})} \int_{\bar{s}}^{s^{\max }} s f_{1}(s) d s}{F_{0}(\bar{s})+\frac{f_{0}(\bar{s})}{f_{1}(\bar{s})}\left(1-F_{1}(\bar{s})\right)} .
$$

It can be checked that $p^{o p t, A D}(\bar{s})$ is a strictly decreasing function with $p^{o p t, A D}\left(s^{\min }\right)=E_{1}[s]$ and $p^{o p t, A D}\left(s^{\max }\right)=E_{0}[s]$.

Note also that the market clearing condition (34) can be rearranged as:

$$
p=p^{m c, A D}(\bar{s}) \equiv n_{1}+\int_{s^{\min }}^{\bar{s}} s \frac{f_{0}(s)}{F_{0}(\bar{s})} d s-n_{0} \frac{f_{0}(\bar{s})}{f_{1}(\bar{s})}\left(\frac{1-F_{1}(\bar{s})}{F_{0}(\bar{s})}\right) .
$$

In addition, the function $p^{m c, A D}(\cdot)$ is strictly increasing with $\lim _{\bar{s} \rightarrow s^{\min }} p^{m c, A D}(\bar{s})=-\infty$ and $p^{m c, A D}\left(s^{\max }\right)=n_{1}+E_{0}[s]$. It follows that there exists a unique $\left(p, \bar{s}^{*}\right)$ characterized as the intersection of $p^{o p t, A D}(\cdot)$ and $p^{m c, A D}(\cdot)$. Moreover, the solution satisfies $p \in\left(E_{0}[s], E_{1}[s]\right)$ and $\bar{s}^{*} \in\left(s^{\min }, s^{\max }\right)$. Given $\bar{s}^{*}$, traders' returns are given by $R_{0}^{*}=R_{0}\left(\bar{s}^{*}\right) \in\left(1, \frac{f_{0}\left(s^{\min }\right)}{f_{1}\left(s^{\min }\right)}\right)$ and $R_{1}^{*}=R_{1}\left(\bar{s}^{*}\right) \in\left(1, \frac{f_{1}\left(s^{\max }\right)}{f_{0}\left(s^{\min }\right)}\right)$. In view of the above analysis, this solution corresponds to the essentially unique AD equilibrium, completing the proof.

Example 3 (Effect of Richer Contracts on the Asset Price). Consider the state space $\mathcal{S}=[0,1]$. Suppose pessimists and optimists have the prior belief distributions $F_{0}$ and $F_{1}$ with density functions:

$$
f_{0}(s)=1 \text { for each } s \in \mathcal{S}, \quad f_{1}(s)=\left\{\begin{aligned}
7 / 8, & \text { if } s \in[0,3 / 4) \\
11 / 8, & \text { if } s \in[3 / 4,1]
\end{aligned}\right.
$$

which satisfy assumption (MLRP) with weak inequalities. Let $p^{\text {debt }}$ denote the equilibrium price with simple debt contracts (characterized in Theorem 3) and $p^{A D}$ denote the AD equilibrium price (characterized in Theorem 8). Suppose optimists' endowment is given by $n_{1}=0.4$, and consider two cases that differ in pessimists' endowment.

Case (i). Suppose pessimists have a relatively small endowment, i.e., $n_{0}=0.2$. Then, $p^{\text {debt }}=0.537$ and $p^{A D}=0.547=E_{1}[s]$. In particular, the asset price in the $A D$ equilibrium is higher.

Case (ii). Suppose pessimists have a larger endowment, i.e., $n_{0}=1$. Then, $p^{\text {debt }}=0.537$ and $p^{A D}=0.5=E_{0}[s]$. In particular, the asset price in the $A D$ equilibrium is lower.

Hence, richer contracts have an ambiguous effect on the asset price.

\section{References}

Acemoglu, D., V. Chernozhukov, and M. Yildiz (2009), "Fragility of Asymptotic Agreement under Bayesian Learning," working paper, MIT. 
Acharya V., D. Gale, and T. Yorulmazer (2009), "Rollover Risk and Market Freezes," NBER Working Paper, 15674.

Adrian T. And H. S. Shin (2010), "Liquidity and Leverage," Journal of Financial Intermediation, forthcoming.

Allen, F., S. Morris, And A. Postlewaite (1993), "Finite Bubbles with Short Sale Constraints and Asymmetric Information," Journal of Economic Theory, 61, p.206-29.

Blume L. And D. Easley (2006), "If You're so Smart, Why Aren't You Rich? Belief Selection in Complete and Incomplete Markets," Econometrica, 74, p.929-966.

Brunnermeier, M. K. And L. H. Pedersen (2009), "Market Liquidity and Funding Liquidity," Review of Financial Studies, Vol. 22(6), p.2201-2238.

Brunnermeier, M. K. And Y. Sannikov (2011), "A Macroeconomic Model with a Financial Sector," working paper, Princeton University.

Burnside, C., M. Eichenbaum and S. Rebelo (2011), "Understanding Booms and Busts in Housing Markets," NBER working paper, No. 16734.

CAo D. V. (2009), "Collateral Shortages, Asset Price and Investment Volatility with Heterogeneous Beliefs," working paper.

Case, K. and R. Shiller, (2003), "Is There a Bubble in the Housing Market?" Brookings Papers on Economic Activity, 2, p.299-362.

Chen, J., H. Hong, And J. C. Stein (2002), "Breadth of Ownership and Stock Returns," Journal of Financial Economics, Vol. 66, No. 2-3, p.171-205.

Copeland, A., A. Martin, M. Walker (2011), "Repo Runs: Evidence from the Tri-party Repo Market," Federal Reserve Bank of New York, working paper.

D'Avolio, G. (2002), "The Market for Borrowing Stock," Journal of Financial Economics, Vol. 66, p.271-306.

Diether, K., C. Malloy, And A. Scherbina (2002), "Differences of Opinion and the Cross Section of Stock Returns," Journal of Finance, Vol. 57, p.2113-2141.

Duffie, D. (1996), "Special Repo Rates," Journal of Finance, 51, p.493-526.

Duffie, D., N. Garleanu, and L. H. Pedersen (2002), "Securities Lending, Shorting, and Pricing," Journal of Financial Economics, Vol. 66, p.307-339.

Fostel A. And J. Geanakoplos (2008), "Leverage Cycles and the Anxious Economy," American Economic Review, Vol. 98:4, p.1211-1244.

(2011), "Endogenous Leverage: VaR and Beyond," Yale University working paper. (2012), "Tranching, CDS and Asset Prices: Bubbles and Crashes," AEJ: Macroeconomics, 2012, 4(1), p.190-225.

Friedman M. (1953), Essays in Positive Economics, University of Chicago Press, Chicago, IL.

Gale, D. and M. Hellwig (1985), "Incentive-compatible Debt Contracts: The One-period Problem," Review of Economic Studies, 52, p.647-663.

Garleanu N. And L. H. Pedersen (2011), "Margin-based Asset Pricing and Deviations From the Law of One Price," Review of Financial Studies, forthcoming.

Geanakoplos, J. (1997), "Promises, Promises," in The Economy as an Evolving Complex System II, ed. by Arthur, B., S. Durlauf, and D. Lane, p.285-320. Addison-Wesley.

(2003): "Liquidity, Default and Crashes: Endogenous Contracts in General Equilibrium," in Advances in Economics and Econometrics: Theory and Applications II, Econometric Society Monographs: Eighth World Congress, ed. by Dewatripont, M., L. P. Hansen, and S. J. Turnovsky, Cambridge University Press, vol. 2, p.170-205.

(2010): "The Leverage Cycle," in Acemoglu D., K. Rogoff, and M. Woodford, eds., NBER Macroeconomics Annual, p.1-65. 
Geanakoplos, J. and W. Zame (2009), "Collateralized Security Markets," unpublished, earlier versions 1997, 2002, 2005.

Gorton, G. And A. Metrick (2011), "Securitized Banking and the Run on Repo," Journal of Financial Economics, forthcoming.

Gromb, D. And D. Vayanos (2002), "Equilibrium and Welfare in Markets with Financially Constrained Arbitrageurs," Journal of Financial Economics, 66, p.361-407.

HARRISOn, J. M. AND D. M. KREPS (1978), "Speculative Investor Behavior in a Stock Market with Heterogeneous Expectations," Quarterly Journal of Economics, 92, p.323-36.

Hart, O. And J. Moore (1994), "A Theory of Debt Based on the Inalienability of Human Capital," Quarterly Journal of Economics, 109, p.841-79.

He Z. And W. Xiong (2011), "Debt Financing in Asset Markets," American Economic Review Papers and Proceedings, forthcoming.

Holmstrom, B. And J. Tirole (1997), "Financial Intermediation, Loanable Funds, and the Real Sector," Quarterly Journal of Economics, 92, p.663-91.

Hong, H. And D. Sraer (2011), "Speculative Betas," Princeton University.working paper. Jones, C. M. and O. A. Lamont (2001), "Short Sale Constraints and Stock Returns," Journal of Financial Economics, Vol. 66, No. 2-3, p.207-239.

Jurek, J. And E. Stafford (2011), "Crashes and collateralized lending," Princeton University working paper.

Kiyotaki, N. And J. Moore (1997), "Credit Cycles," Journal of Political Economy,Vol.105 (2), p.211-248.

Krishnamurthy, A., S. Nagel, and D. Orlov (2011), "Sizing Up Repo," working paper.

Lakonishok, J., Shleifer A., And R. Vishny (1994), "Contrarian Investment, Extrapolation, and Risk," Journal of Finance, 49, p.1541-1578.

Lamont, O. (2004), "Short Sale Constraints and Overpricing," The Theory and Practice of Short Selling, ed. F. J. Fabozzi.

Lamont, O. And J. C. Stein (2004), "Aggregate Short Interest and Market Valuation," American Economic Review Papers and Proceedings, Vol.94, p.29-32.

Lewis, M. (2010), The Big Short: Inside The Doomsday Machine, W.W. Norton and Company, New York, NY.

Miller, E. (1977), "Risk, Uncertainty, and Divergence of Opinion," Journal of Finance, Vol. 32, No. 4, p.1151-1168.

Morris, S. (1995), "The Common Prior Assumption in Economic Theory," Economics and Philosophy, 11 p.227-253.

(1996), "Speculative Investor Behavior and Learning," Quarterly Journal of Economics, Vol. 111, p.1111-1133.

Myers, S. AND N. S. Majluf (1984), "Corporate Financing and Investment Decisions when Firms have Information that Investors Do Not Have," Journal of Financial Economics, Vol. 13, No. 2, p.187-221.

Ofek, E. And M. Richardson (2003), "DotCom Mania: The Rise and Fall of Internet Stock Prices," Journal of Finance, Vol. 58, p.1113-1137.

Prescott, E. C. And R. Townsend (1984a), "General Competitive Analysis in an Economy with Private Information," International Economic Review, 25, p.1-20.

(1984b), "Pareto Optima and Competitive Equilibria with Adverse Selection and Moral Hazard," Econometrica, 52, p.21-45.

Reinhart, C. M. and K. S. Rogoff (2008), "Is the 2007 U.S. Subprime Crisis So Different? An International Historical Comparison," American Economic Review, Vol. 98, No. 2, p.339-344. 
Sandroni, A. (2000), "Do Markets Favor Agents Able to Make Accurate Predictions?" Econometrica, Vol. 68, p.1303-1342.

Scheinkman, J. A. And W. Xiong (2003), "Overconfidence and Speculative Bubbles," Journal of Political Economy, Vol. 111, p.1183-1219.

Shleifer, A. And R. Vishny (1992), "Liquidation Values and Debt Capacity: A Market Equilibrium Approach," Journal of Finance, 47, p.1343-1366.

(1997), "The Limits of Arbitrage," Journal of Finance, 52, p.35-55.

Shiller, R. J. (2005), Irrational Exuberance, 2nd Edition, Princeton University Press, Princeton, NJ.

Simsek, A. (2010), Essays on Uncertainty in Economics, Ph.D. Thesis, MIT Department of Economics.

Townsend, R. (1979), "Optimal Contracts and Competitive Markets with Costly State Verification," Journal of Economic Theory, 21, p.265-293.

Yu, J. (2011), "Disagreement and Return Predictability of Stock Portfolios," Journal of Financial Economics, 99, p.162-183. 